\title{
Dynamics of non-classical interval exchanges
}

\author{
Thesis by \\ Vaibhav S Gadre \\ In Partial Fulfillment of the Requirements \\ for the Degree of \\ Doctor of Philosophy
}

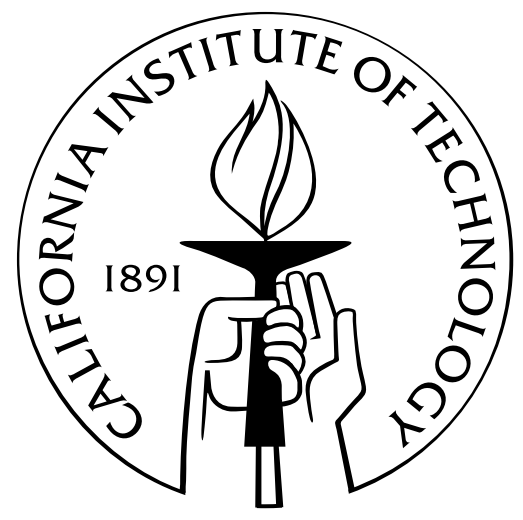

California Institute of Technology

Pasadena, California

2010

(Submitted April 21, 2010) 
(C) 2010

Vaibhav S Gadre

All Rights Reserved 


\section{Acknowledgements}

This thesis would not exist without the support of my advisor, Prof. Nathan Dunfield. Through all these years of working with him, Nathan has been incredibly generous with his time. The number of occasions I have rambled on vaguely about a problem are too many to recount. Yet, Nathan never lost patience, and provided encouragement and direction every single time.

I am immensely grateful to Chris Leininger, Joseph Maher, Saul Schleimer and Steve Kerckhoff. At UIUC, Chris has been like a second advisor to me. The numerous and very fruitful conversations I have had with Joseph, Saul and Steve have been instrumental in clarifying some of the key ideas. I thank Chia-yen Tsai for her profusion of ideas on a joint work in progress. I thank Susan Parker for mentoring me at the time when I first started teaching.

I really cherish the openness of the mathematical community. I would like to thank everyone with whom I had a chance to talk about mathematics, especially Danny Calegari, Daniel Ruberman, and Sharad Kanetkar.

The mathematics departments at Caltech and UIUC have been my home for the past six years. I thank the faculty and the graduate students at both these places for making it so interesting.

I would like to thank all my friends for the most enjoyable time I have had in their company, especially Imran Sheikh, Vikram Gavini and Pinkesh Patel. I thank my wife's parents, her brother Sameer and his wife Manik for their support. I also thank Kalyani and Gaurav, and Nirmala mavshi and 
Balmohan kaka for the encouragement they provided at various times.

None of this was possible without the support of my parents. From a young age, they instilled in me a curiosity and an enthusiasm for science and mathematics. In a high school system which values grades over understanding, I was not adept at getting very good scores. They bore the social pressure that resulted with great fortitude. My mother sustained the courage and wisdom to help me through academic and career choices that went against conventional wisdom. I thank them with all my heart.

Special thanks to my wife, Sonali. Her love and friendship give meaning to everything. 


\section{Abstract}

Train tracks with a single vertex are a generalization of interval exchange maps. Here, we consider non-classical interval exchanges: complete train tracks with a single vertex. These can be studied as a dynamical system by considering Rauzy induction in this context. This gives a refinement process on the parameter space similar to Kerckhoff's simplicial systems. We show that the refinement process gives an expansion that has a key dynamical property called uniform distortion. We use uniform distortion to prove normality of the expansion. Consequently, we prove an analog of Keane's conjecture: almost every non-classical interval exchange is uniquely ergodic. In the concluding chapter, we state an application of the main results of the thesis to a question about harmonic measures on the Thurston boundary of Teichmüller space coming from finitely supported random walks on the mapping class group. 


\section{Contents}

Acknowledgements

Abstract $\quad$ V

1 Introduction 1

1.1 Outline of the thesis . . . . . . . . . . . . . 7

2 Non-classical Interval Exchanges $\quad 9$

3 Rauzy Induction $\quad 13$

3.1 Rauzy induction . ................... 13

3.2 Rauzy diagram ....................... 15

$\begin{array}{lll}4 & \text { Dynamics } & 17\end{array}$

4.1 Description of the parameter space . . . . . . . . . . . 17

4.2 Irreducibility $\ldots \ldots \ldots \ldots$

4.3 Iterations of Rauzy induction . . . . . . . . . . . . . 20

5 Kerckhoff's Approach: Some Examples 25

5.1 Classical interval exchange with two bands . . . . . . . . . . 25

5.2 The general approach . . . . . . . . . . . . . . . 27

5.3 Issues with non-classical interval exchanges . . . . . . . . . 28

6 Jacobian of the Restriction $\quad 34$ 
$\begin{array}{lll}7 & \text { Measure Theory } & 39\end{array}$

7.1 Measure theory of projective linear maps . . . . . . . . . . . . 39

7.2 Evaluating measures . . . . . . . . . . . . . . 40

7.2 .1 Wedges .................. 40

7.2.6 Half-space intersections . . . . . . . . . . . . . . 41

8 Proof of Theorem 4.3.2 44

$\begin{array}{lll}9 & \text { Normality } & 68\end{array}$

$\begin{array}{ll}10 \text { Unique Ergodicity } & 70\end{array}$

11 Rauzy Map on the Parameter Space $\quad 74$

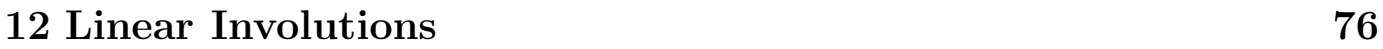

$\begin{array}{ll}13 \text { Applications and Future Research } & 78\end{array}$

13.1 Harmonic measures on $\mathcal{P} \mathcal{M} \mathcal{F}$. . . . . . . . . . . . . 78

13.2 Future directions . . . . . . . . . . . . . . . 80

$\begin{array}{lr}\text { Bibliography } & 81\end{array}$ 


\section{List of Figures}

1.0.1 A classical interval exchange . . . . . . . . . . . . . . 2

2.0.1 A non-classical interval exchange on a 4-punctured sphere . . . 11

3.1 Rauzy induction ... . . . . . . . . . . . . . 13

5.3.1 Splitting sequence . . . . . . . . . . . . . . . . 29

$5.3 .2 W$ in $\Delta \ldots \ldots \ldots \ldots$

$5.3 .3 W(Q)$ in $\Delta(Q) \ldots \ldots \ldots \ldots$

5.3.4 Projection of $\mathrm{W}(\mathrm{Q}) \ldots \ldots \ldots \ldots$

5.3.5 Band 1 splits Band 2 ..................... 32

6.0.5 Schematic picture . . . . . . . . . . . . . 37

$7.2 .3 \Delta_{R}(\alpha, \beta) \ldots \ldots \ldots \ldots \ldots \ldots \ldots$

8.0.9 Schematic Picture . . . . . . . . . . . . . . 50

$8.0 .18 \gamma$ splits $\sigma \ldots \ldots \ldots \ldots \ldots \ldots \ldots$ 


\section{Chapter 1}

\section{Introduction}

In this thesis, we are interested in the dynamical properties of non-classical interval exchanges. The idea behind classical interval exchange maps is to partition an interval $I$ into $d$ subintervals, permute these subintervals and then glue them back, preserving their orientation, to get back the original interval I. The result is a Lebesgue measure preserving map from $I$ to itself which we call a classical i.e.m. for short. The data that completely determines a classical i.e.m. is, first, the lengths of the subintervals and second, the permutation used for gluing. There is a way to draw these maps pictorially:

Draw the original interval $I$ horizontally in the plane and then thicken it vertically to get two copies of $I$, the top and the bottom. The bottom interval is divided into $d$ subintervals with the prescribed lengths. The top interval is also divided into $d$ subintervals but the permutation is incorporated into the subdivision to decide the lengths. Each subinterval on the bottom pairs off, by the permutation, with a subinterval on the top with the same length. Join these subintervals by a band of uniform width equal to their common length.

As an example, a classical interval exchange with 2 subintervals is shown in Figure 1.0.1. 
Train tracks enter the picture as follows: To each band we can associate a central edge that runs along the length of the band from the bottom to the top. If we collapse the bands to these central edges and the thickened interval horizontally to a vertical edge and finally collapse the vertical edge, we get a train track with a single vertex. The resulting train track has the property that every outgoing edge is incoming on the other side of the vertex. A generalization of interval exchanges can



Figure 1.0.1: A classical interval exchange be defined by relaxing this last property. The picture associated will be of a thickened interval with bands, in which orientation-reversing bands that run from bottom to bottom or top to top are allowed. Here, we shall consider tracks that are recurrent. This implies that if there are orientation-reversing bands on one side of the thickened interval, then there is at least one orientationreversing band on the other side. Moreover, we assume that the abstract train track considered admits an embedding into a surface with non-positive Euler characteristic as a complete train track. A necessary condition for this is that there is at least one orientation-reversing band on each side. We shall call the thickened interval with bands associated to such a train track, a non-classical interval exchange. The Lebesgue measure on the interval is invariant under a non-classical interval exchange; the precise meaning of this will be outlined in Chapter 2.

For classical i.e.m.'s Keane conjectured that if the reshuffle permutation is irreducible, then for almost all widths of the bands, the associated classical i.e.m. is uniquely ergodic. In particular, the Lebesgue measure on the interval is the only invariant probability measure. This conjecture was first proved, 
independently, by Masur [14] and Veech [19]. See also Rees [17] and Zorich [21]. A key tool was Rauzy induction, alternatively called splitting: this is the process of inducing a classical i.e.m. on a suitable subinterval by considering the first return map. Veech [19] used this to develop a continued fraction expansion for classical i.e.m.'s. When the classical i.e.m. has two bands, it is the same as the continued fraction expansion of the ratio of the widths of the bands. Veech showed that this expansion had most of the nice properties of the classical Gauss map. He derived unique ergodicity as a consequence of this.

A similar conjecture was also made for transverse measures for measured foliations on a Riemann surface. Masur proved this conjecture along with the original Keane conjecture in [14]; his approach relied on techniques in Teichmüller theory and applied to both contexts. Later, Kerckhoff [13] developed a different and more elementary approach that does not rely on Teichmüller theory, to include both conjectures under a unified setup. In his formulation, he encoded Rauzy induction as a refinement process on the parameter space associated to a classical i.e.m. For measured foliations, Rauzy induction generalizes to an induction process called splitting train tracks. So the refinement process makes sense also for the parameter space associated to a complete train track.

For a classical i.e.m. with $d$ bands, the parameter space at each stage of the process can be identified with the standard simplex of dimension $(d-1)$. Rauzy induction splits this simplex into two halves, each a $(d-1)$-simplex itself. A point in the original simplex picks out the half in which it lies. In addition, there is a map from the new parameter space to the starting parameter space. It identifies the standard simplex with the half that is picked out. After this identification, the simplex given by the new parameter space becomes the 
stage to which the induction is applied and the process continues. Associated to each stage given by a finite sequence of inductions, there is a map from the current parameter space back into the starting parameter space. This map is defined iteratively as a composition of the maps associated to individual steps in the sequence.

It turns out that, almost surely, iterations give an infinite expansion consisting of nested simplices on the parameter space. It can be shown that there is a canonical identification of the infinite intersection of these nested simplices with the set of invariant probability measures. The refinement process can be defined purely abstractly; Kerckhoff called this a simplicial system and the resulting expansion, a simplicial expansion. He showed that if a simplicial system satisfies a combinatorial condition called the absence of isolated blocks, then for almost every initial point in the starting simplex, the resulting simplicial expansion is normal: almost surely, every finite sequence of inductions that can occur in the expansion, does occur infinitely often. Finally, normality and the existence of finite sequences which shrink the diameter by a definite amount ensure that, almost surely, the nested sequence of simplices actually nests down to a point. If the reshuffle permutation of a classical i.e.m. is irreducible then Kerckhoff showed that its simplicial system has no isolated blocks. Consequently, almost surely, the expansion nests down to a point and so there is a unique invariant probability measure. This means one has unique ergodicity.

For complete train tracks, the parameter space is the set of possible weights carried by the train track, normalized so that the sum of weights is one. So it is a convex subset of the ambient simplex cut out by the switch conditions of the train track. It has codimension in the simplex equal to the number of switch conditions. For a non-classical interval exchange with $d$ bands, there is 
a single switch condition. So the parameter space is of codimension 1 in the standard $(d-1)$-dimensional simplex. The main theorem we show here is:

Theorem 1.0.2 (Normality). For non-classical interval exchanges, almost surely, the expansion is normal, i.e., every finite non-transient sequence that can occur in the expansion, does occur infinitely often.

As we will see in Chapter 4, combinatorially, there are finitely many subsets inside the standard $(d-1)$-simplex which can be the parameter space for a non-classical interval exchange with $d$ bands. We shall call each such subset a configuration space and denote them by $W_{r}$. For all the subsequent discussion, we fix a combinatorial type $\pi_{0}$ for the initial non-classical interval exchange and call it the starting stage. The starting stage is assumed to be irreducible and in a sink of the Rauzy diagram (see Chapter 4 for the precise definitions). Let $W_{0}$ be the configuration space at $\pi_{0}$. As shall be explained in Chapter 4 , for almost every point in $W_{0}$, repeated Rauzy induction defines an infinite expansion. Let $\pi^{\prime}$ be a subsequent stage obtained from $\pi_{0}$ by a finite sequence of inductions. Let $W^{\prime}$ be the configuration space at $\pi^{\prime}$. The associated map $f^{\prime}$ relates the new widths of the bands to the starting widths in $\pi_{0}$, by giving a diffeomorphism from $\Delta$ onto a subset of itself, such that $f^{\prime}\left(W^{\prime}\right)=W_{0} \cap f^{\prime}(\Delta)$. Fix a constant $C>1$. The stage $\pi^{\prime}$ is $C$-uniformly distorted if, for any pair of points $\mathbf{y}, \overline{\mathbf{y}}$ in $W^{\prime}$, the Jacobian of $f^{\prime}$ thought of as a map from $W^{\prime}$ to $W_{0}$, satisfies

$$
\frac{1}{C}<\frac{\partial\left(f^{\prime}\right)(\mathbf{y})}{\partial\left(f^{\prime}\right)(\overline{\mathbf{y}})}<C
$$

Let $\pi$ be any stage obtained from $\pi_{0}$ by a finite sequence of inductions. Let $W$ be the configuration space at $\pi$ and $f$ be the associated map. The main technical theorem required to prove Theorem 1.0.2 is the uniform distortion theorem, stated below: 
Theorem 1.0.3 (Uniform Distortion). There exists a constant $C>1$, independent of the stage $\pi$, such that, for almost every $\mathbf{x} \in f(W)$, the associated expansion of $\mathbf{x}$ after $\pi$ has some stage $\pi^{\prime}$, depending on $\mathbf{x}$, such that $\pi^{\prime}$ is $C$ uniformly distorted. Moreover, if $\pi_{0}$ is in a sink of the Rauzy diagram, then $\pi^{\prime}$ can be assumed to be combinatorially the same as $\pi_{0}$.

Uniform distortion implies that the relative probability of a permissible finite sequence occurring right after a uniformly distorted stage is roughly the same as the probability that an expansion begins with that sequence. Normality is a straightforward consequence of this phenomena.

This approach was originally outlined by Kerckhoff in [13]. For classical i.e.m.'s, he showed uniform distortion and consequently normality and unique ergodicity. However, for complete train tracks, there are two issues in making this work. Firstly, the probability of a particular split is the ratio of the volume of the part of the configuration space that is inside the smaller simplex picked out by the split to the volume of the entire configuration space. As we shall see in Chapter 5, this ratio can be very different from the proportion of the volumes of the ambient simplices. Secondly, splitting sequences of complete train tracks can have isolated blocks. These issues leave the proof of unique ergodicity for measured foliations in [13] incomplete.

We do not know how to fix this for general complete train tracks. Nevertheless, we settle these issues for non-classical interval exchanges here, by proving Theorems 1.0.3 and 1.0.2. As a consequence we also show in Theorem 11.0.1 that the map on the parameter space given by Rauzy induction is ergodic. Finally, using the irreducibility criteria for linear involutions by Boissy and Lanneau, we indicate how to prove the theorems for geometrically irreducible linear involutions. 


\subsection{Outline of the thesis}

In Chapter 2, we begin with the definition of a classical i.e.m. and see how its pictorial interpretation leads naturally to the definition of a non-classical interval exchange. In Chapter 3, we define Rauzy induction. This defines a directed graph associated to non-classical interval exchange with a fixed number of bands. For classical i.e.m., this graph is called a Rauzy diagram. We continue to call it a Rauzy diagram for non-classical interval exchanges. In Chapter 3.2, we describe the key differences between the Rauzy diagram for a non-classical interval exchange and the classical Rauzy diagram and their relevance to Theorems 1.0.2 and 1.0.3. In Chapter 4, we show how Rauzy induction defines a refinement process on the parameter space. This associates infinite expansions by iterated induction to almost every point in the parameter space. We then formulate the main theorem, Theorem 4.3.2, which is the more precise statement of Theorem 1.0.3. In Chapter 5, we illustrate by an example the main ideas of Kerckhoff's original proof for classical i.e.m. We also provide an example of a splitting sequence of non-classical interval exchange with 4 bands that illustrates a key issue which makes the proof for non-classical interval exchanges more intricate. This issue arises from the fact that the parameter space for non-classical interval exchanges is a codimension one subset of the ambient simplex. Resolving the issue requires understanding the Jacobian of the restriction of the projective linear map to this subset instead of considering the full simplex. This is done in Chapter 6. In Chapter 7 , we recall the main results in the measure theory of projective linear maps with non-negative entries from a standard simplex into itself, and use it to compute the measures of some standard subsets that we will encounter in the later section. Chapter 8 gives the detailed proof of Theorem 4.3.2. In 
Chapter 9, we prove normality from Theorem 4.3.2. Finally in Chapter 10, we use normality to show unique ergodicity. As a consequence, we show in Chapter 11 that the Rauzy map on the parameter space is ergodic. Finally, in Chapter 12, we indicate why the theorems generalize to geometrically irreducible linear involutions. In the concluding chapter, we state an application of the main results of the thesis to a question about harmonic measures on the Thurston boundary of Teichmüller space coming from finitely supported random walks on the mapping class group. 


\section{Chapter 2}

\section{Non-classical Interval Exchanges}

For a precise definition of a classical interval exchange, see Yoccoz [20]. Here we will focus on representing a classical i.e.m. pictorially.

Let $S$ denote the set $\{1,2, \ldots, d\}$. A classical i.e.m. is determined by the lengths $\left(\lambda_{\alpha}\right), \alpha \in S$ of the subintervals and a permutation $\pi$ of $S$. A classical i.e.m. is represented pictorially as follows: In the plane, draw the interval $I=\left[0, \sum_{\alpha} \lambda_{\alpha}\right)$ along the horizontal axis and then thicken it slightly in the vertical direction to get two copies, $I \times(\delta)$ and $I \times(-\delta)$. Call them top interval and bottom interval respectively. Subdivide the bottom interval into $d$ sub-intervals with lengths $\lambda_{1}, \lambda_{2}, \ldots, \lambda_{d}$ from left to right. Subdivide the top interval into $d$ sub-intervals with lengths $\lambda_{\pi^{-1}(1)}, \lambda_{\pi^{-1}(2)}, \ldots, \lambda_{\pi^{-1}(d)}$ from left to right. Now join each subinterval on the bottom to the corresponding subinterval on the top by a band of uniform width $\lambda_{\alpha}$. To determine the image of a point $x \in I$ under the i.e.m., pick the subinterval on the bottom in which $x$ lies and flow $x$ along the band to the top. The only ambiguity in the definition occurs at the endpoints of the subintervals because they can be thought of as belonging to two adjacent bands. This is removed by requiring the endpoint flow along the band that lies to the right.

One can construct a train track from this picture. Recall that a train track is a 1-dimensional CW complex in which there is a common line of tangency to 
all the 1-dimensional branches that join at a 0-dimensional switch. This splits the set of branches incident on a switch into two subsets, one of which will be called the set of outgoing edges and the other the set of incoming edges. In addition, one assigns non-negative weights to the branches so that the sum of the weights of the outgoing branches at a switch is equal to the sum of the weights of the incoming branches. In our picture, each band has a central edge which joins the midpoint of the subinterval on the bottom to the midpoint of the corresponding subinterval on the top. Retract each band to its central edge and the thickened interval to a vertical edge. It is clear that this can be done in a way such that the edges associated to the bands share a vertical line of tangency on each side of this vertical edge. Finally, retract the central vertical edge to a point. The result is a train track with a single vertex. It has the property that every outgoing edge from the bottom is incoming from the top and vice versa. Also, assign the width of the associated band as the weight on the branch. It is immediate that this assignment of weights is consistent with the switch condition.

The first step towards defining non-classical interval exchanges is to relax the constraint that every outgoing edge from the bottom be incoming from the top and vice versa, i.e., to allow bands that run from bottom to bottom or top to top. We call such bands orientation-reversing because the flow along such a band reverses the orientation of the subinterval.

A train track is recurrent if there is an assignment of weights satisfying the switch conditions, such that all the weights are strictly positive. Here, it implies that if there are orientation-reversing bands on one side then there has to be at least one orientation-reversing band on the other side. The interval exchange that we get from this picture shall be called a recurrent interval exchange. A train track is transversely recurrent if for every branch of the 
train track there is an essential simple closed curve hitting the train track efficiently (i.e., the complement of the union of the track and the curve has no bigons) such that the curve intersects the branch. A train track is maximal if it is not a proper subtrack of any other train track. A recurrent and transversely recurrent train track is said to be birecurrent. A maximal birecurrent train track is said to be a complete train track.

We define a non-classical interval exchange to be an interval exchange whose abstract train track admits an embedding into some oriented surface with non-positive Euler charac-

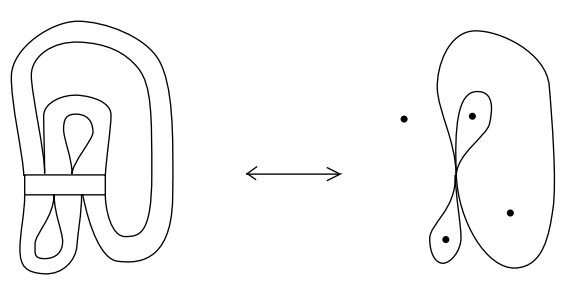
teristic, as a complete train track on the surface. For an interval exchange Figure 2.0.1: A non-classical interval with more than 2 bands to be non- exchange on a 4-punctured sphere classical, it is sufficient that the complementary regions are either ideal triangles or once-punctured monogons. Maximality and recurrence of the underlying track follow immediately and transverse recurrence follows by an easy application of Corollary 1.3.5 in [16].

It follows that for recurrence of a non-classical interval exchange, it is necessary that there is at least one orientation band on each side. An example of a non-classical interval exchange is shown in Figure 2.0.1; the train track on the right is a complete train track on a sphere with 4 punctures. In the figure the punctures are indicated by the dots.

It must be pointed out that once there are orientation-reversing bands, there is no actual map from the interval $I$ to itself. In a classical i.e.m. one can orient the bands to flow from the bottom to the top. This is no longer possible once there are orientation-reversing bands. The point is that the non-classical 
interval exchanges carry vertical measured foliations on the Riemann surface that are associated to quadratic differentials, whereas the classical i.e.m.'s carry vertical measured foliations that are associated to abelian differentials.

Here, we shall work with labeled non-classical interval exchanges. A labeling is a bijection from $S$ to the set of bands. Non-classical interval exchanges with the same exchange combinatorics but different labeling shall be regarded as different. We shall denote a non-classical interval exchange by $T=\left(\left(\lambda_{\alpha}\right), \pi\right)$, where $\pi$ shall denote the exchange combinatorics and $\left(\lambda_{\alpha}\right)$ specifies the widths of the bands according to the labeling. We call the positions on the top and bottom that are rightmost on the intervals the critical positions. 


\section{Chapter 3}

\section{Rauzy Induction}

\subsection{Rauzy induction}

We shall now describe Rauzy induction. Since the underlying picture is that of an interval with bands, Rauzy induction for a non-classical interval exchange is exactly like Rauzy induction for a classical i.e.m. Iterations of Rauzy induction on a classical i.e.m. give an expansion analogous to the continued fraction expansion. In fact, when the classical i.e.m. has just two bands, the expansion by Rauzy induction is the same as the continued fraction expansion of the ratio of the widths of the two bands.

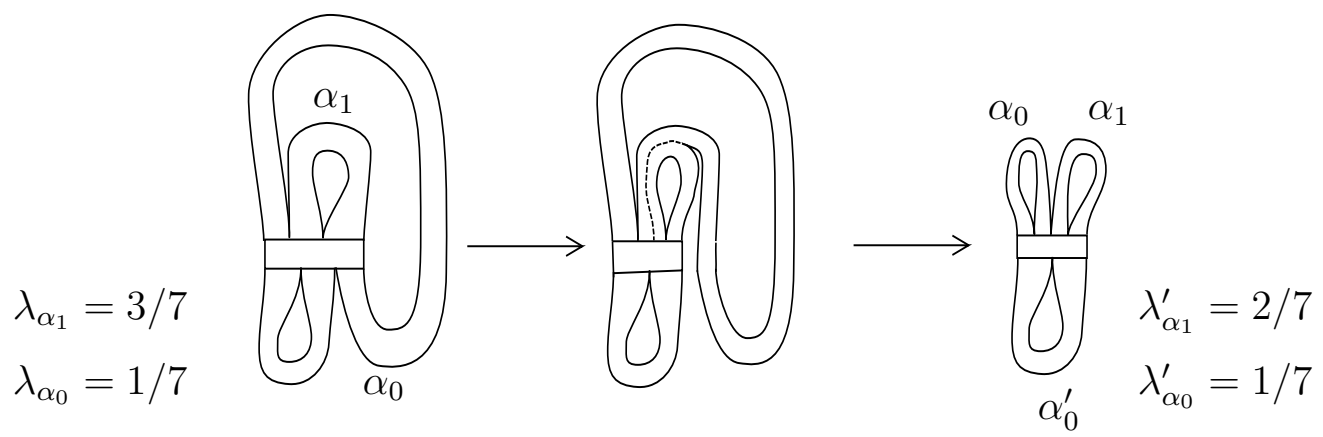

Figure 3.1.1: Rauzy induction

Suppose $T$ is a non-classical interval exchange. Let $\alpha_{0}$ and $\alpha_{1}$ be the labels of the bands in the critical positions with $\alpha_{1}$ on the top. First, suppose that 
$\lambda_{\alpha_{1}}>\lambda_{\alpha_{0}}$. Then we slice as shown in Figure 3.1.1 till we hit the original interval for the first time. The $\alpha_{1}$ band remains in its critical position, but typically a band with a different label $\alpha_{0}^{\prime}$ moves into the other critical position. Furthermore, the new width of $\alpha_{1}$ is $\lambda_{\alpha_{1}}-\lambda_{\alpha_{0}}$. All other widths remain unchanged. If $\lambda_{\alpha_{1}}<\lambda_{\alpha_{0}}$ instead, we slice in the opposite direction, which in Figure 3.1.1 would be the analogous operation after flipping the picture about the horizontal axis. In either case, we get new non-classical interval exchanges with combinatorics and widths as described above. The operation we just described is called Rauzy induction. The non-classical interval exchange we get by Rauzy induction carries an induced labeling. Since Rauzy induction is represented pictorially by one band being split by another, it's also called a split. This is consistent with the notion of a split in the context of train-tracks; if the interval exchange is thought of as a train track then Rauzy induction is the same as splitting the train track. Iterations of Rauzy induction are called splitting sequences.

For a classical i.e.m., Rauzy induction is the first return map under $T$ to the interval $I^{\prime}=\left[0, \sum_{\alpha \neq \alpha_{0}} \lambda_{\alpha}\right)$ in the first instance and $I^{\prime}=\left[0, \sum_{\alpha \neq \alpha_{1}} \lambda_{\alpha}\right)$ in the second.

Let $M_{\alpha \beta}$ be the $d \times d$-matrix with the $(\alpha, \beta)$ entry 1 and all other entries 0 . The relationship between the old and new width data can be expressed by

$$
\lambda=E \lambda^{\prime}
$$

where the matrix $E$ has the form $E=I+M$. In the first instance of the split, when $\lambda_{\alpha_{1}}>\lambda_{\alpha_{0}}$, the matrix $M=M_{\alpha_{1} \alpha_{0}}$; in the second instance of the split, when $\lambda_{\alpha_{0}}>\lambda_{\alpha_{1}}$, the matrix $M=M_{\alpha_{0} \alpha_{1}}$. Thus, in either case the matrix $E$ is an elementary matrix, in particular $E \in S L(d ; \mathbb{Z})$. If $B$ is any $d \times d$ matrix 
then in the instance when $\lambda_{\alpha_{1}}>\lambda_{\alpha_{0}}$, the action on $B$ by right multiplication by $E$ has the effect that the $\alpha_{0}$-th column of $B$ is replaced by the sum of the $\alpha_{0}$-th column and $\alpha_{1}$-th column of $B$. We phrase this as: in the split, the $\alpha_{1}$-th column moves $\alpha_{0}$-th column. Similar statements hold when $\lambda_{\alpha_{0}}>\lambda_{\alpha_{1}}$.

One question that remains is what happens when $\lambda_{\alpha_{1}}=\lambda_{\alpha_{0}}$ ? The split is undefined as it stands. As we shall see in Chapter 4, the expansion by splitting repeatedly is infinite, almost surely.

\subsection{Rauzy diagram}

One can construct an oriented graph for non-classical interval exchanges similar to the Rauzy diagram for a classical i.e.m. However, there are some key differences which we now explain.

Construct an oriented graph $\bar{G}$ as follows: the nodes of the graph are combinatorial types $\pi$ of labeled non-classical interval exchanges with $d$ bands. Draw an arrow from $\pi$ to $\pi^{\prime}$, if $\pi^{\prime}$ is a combinatorial type resulting from splitting $\pi$. For each node $\pi$, there are at most two arrows coming out of it. A splitting sequence gives us a directed path in $G$.

It is possible to construct a different oriented graph $G$ in which the nodes are combinatorial types of non-classical interval exchanges with $d$ bands without labeling. The arrows are drawn as before. The graph $G$ is analogous to the reduced Rauzy diagram for irreducible classical i.e.m. See Section 4.2 of Yoccoz [20]. There is an obvious map from $\bar{G}$ to $G$ given by forgetting the labeling.

For irreducible classical i.e.m.'s, each connected component of the Rauzy diagram is strongly connected, i.e., any node can be joined to any other node by a directed path. This implies that the reduced Rauzy diagram is also 
strongly connected.

This is not the case with non-classical interval exchanges. For example, the reduced Rauzy diagram for complete genus-2 interval exchanges is not strongly connected. See Dunfield and Thurston, D. [9] and for the general case Boissy and Lanneau [6]. In Chapter 4, we shall see why this issue is not significant for the measure theory.

In our discussion, we shall always refer to the reduced Rauzy diagram $G$. 


\section{Chapter 4}

\section{Dynamics}

In this section, we analyze the expansion by splitting sequences on the space of widths of the bands. It turns out that the space of invariant probability measures embeds into the space of widths and the iterative refinement process defined by the splitting sequences, in the limit, nests down to the set of invariant probability measures. So the basic idea, due to Kerckhoff, is to show that for almost every non-classical interval exchange, one nests down to a single point.

\subsection{Description of the parameter space}

For all non-classical interval exchanges $T$ sharing the same combinatorics $\pi$, a choice of labeling gives a bijection between the set of bands and the standard basis of $\mathbb{R}^{d}$. Let $\left\{e_{\alpha}\right\}$ denote the standard basis under the bijection. We get a map from the set of such $T$ into $\left(\mathbb{R}_{\geq 0}\right)^{d} \backslash\{0\}$ by thinking of $\left(\lambda_{\alpha}\right)$ as co-ordinates for $e_{\alpha}$. Denote the image by $W$. The normalization $\sum \lambda_{\alpha}=1$, restricts $W$ to lie in the standard $(d-1)$-simplex $\Delta$. The switch condition satisfied by the interval exchanges is the other constraint that points in $W$ satisfy. Let $S_{t}$

and $S_{b}$ be the labels of orientation-reversing bands on the top and the bottom 
respectively. The switch condition is equivalent to

$$
\sum_{\alpha \in S_{t}} \lambda_{\alpha}=\sum_{\alpha \in S_{b}} \lambda_{\alpha}
$$

Thus $W$ is the intersection with $\Delta$ of a codimension 1 subspace of $\mathbb{R}^{d}$. For $\alpha \in S_{t}$ and $\beta \in S_{b}$, let $e_{\alpha \beta}$ be the midpoint of the edge $\left[e_{\alpha}, e_{\beta}\right]$ of $\Delta$ joining the vertices $e_{\alpha}$ and $e_{\beta}$. The subset $W$ is the convex hull of the points $e_{\alpha \beta}$ and $e_{\rho}$ for $\rho \notin S_{t} \cup S_{b}$.

There are finitely many pairs $\left(S_{t}, S_{b}\right)$ of disjoint subsets of $S$. So there are finitely many convex codimension 1 subsets of $\Delta$ that could be $W$. We let $\mathcal{J}$ be the set of possible pairs $\left(S_{t}, S_{b}\right)$ and call the subsets of $\Delta$ the pairs define as configuration spaces. We index elements of $\mathcal{J}$ by $r$ and whenever necessary, index the corresponding configuration space as $W_{r}$.

The full parameter space for a non-classical interval exchange with $d$ bands is a disjoint union of configuration spaces, each of which is given by a combinatorial type and a choice of labeling. The labeling once specified initially induces forward under splitting sequences.

\section{$4.2 \quad$ Irreducibility}

For the initial discussion in this subsection, we only assume that our interval exchanges are recurrent and formulate a notion of irreducibility for them. Subsequently, we show that if we assume that our interval exchanges are complete, i.e., by definition non-classical interval exchanges, then they are irreducible.

Definition 4.2.1. A combinatorial type $\pi$ of a recurrent interval exchange is combinatorially reducible if $S$ can be written as a disjoint union $S_{1} \sqcup S_{2}$ of nonempty subsets such that for all $\alpha \in S_{1}$ and $\beta \in S_{2}$, the ends of $\alpha$ bands 
occur to the left of the ends of $\beta$ bands.

This means that for some choice of widths, the interval $I$ can be cut into two intervals $I_{1}$ and $I_{2}$ such that all the $S_{1}$ bands are incident on $I_{1}$ and all the $S_{2}$ bands are incident on $I_{2}$. Thus, $I_{1}$ and $I_{2}$ define interval exchanges by themselves with $\# S_{1}$ and $\# S_{2}$ bands respectively. So for some choice of widths, the original interval exchange is obtained by concatenating two disjoint interval exchanges with fewer bands.

When does combinatorial reducibility imply measure reducibility? In other words, if $\pi$ is combinatorially reducible as $S=S_{1} \sqcup S_{2}$, does this decompose $I$ into interval exchanges $I_{1}$ and $I_{2}$ for all widths? The answer is straightforward.

Proposition 4.2.2. A combinatorial type $\pi$, combinatorially reducible as $S=$ $S_{1} \sqcup S_{2}$, is measure reducible if and only if all the orientation-reversing bands in $\pi$ are entirely in $S_{1}$ or entirely in $S_{2}$.

Proof. Let $S_{1, b}$ and $S_{1, t}$ denote the set of orientation-reversing bands in $S_{1}$ that are incident on the bottom and top respectively. $\pi$ is measure reducible if and only if the additional constraint

$$
\sum_{\alpha \in S_{1, b}} \lambda_{\alpha}=\sum_{\beta \in S_{1, t}} \lambda_{\beta}
$$

gives the defining equation of $W$ or is vacuous. If the former, then all the orientation-reversing bands of $\pi$ have to be in $S_{1}$. If the latter, then all the orientation-reversing bands of $\pi$ have to be in $S_{2}$.

Definition 4.2.4. A combinatorial type $\pi$ of a recurrent interval exchange is said to be irreducible if it has no measure reduction.

The next proposition tells us that a non-classical interval exchange is always irreducible. 
Proposition 4.2.5. Suppose $\pi$ is the combinatorial type of a non-classical interval exchange. Then $\pi$ is irreducible.

Proof. Suppose that $\pi$ admits a measure reduction $S=S_{1} \sqcup S_{2}$. By the previous proposition, all the orientation-reversing bands in $\pi$ must belong entirely say to $S_{1}$. Mark the point on $I$ that is the common endpoint of both the intervals $I_{1}$ and $I_{2}$. It's clear that one can first slice the train track vertically through this common point to break it into a union of two disjoint tracks and then embed it into the surface. This means that any foliation carried by the embedded copy of the original track is now carried by the embedded copy of the new track. Now one of the complementary regions for the new track is not an ideal triangle. So the foliations cannot be complete, which is a contradiction.

\subsection{Iterations of Rauzy induction}

We shall restrict our initial choice to the set of non-classical interval exchanges with fixed combinatorics $\pi_{0}$ and a fixed labeling. The choice picks out a connected component in the full parameter space. Identify the set of normalized non-classical interval exchanges $T$ compatible with these choices with a configuration space $W_{r_{0}}$ in the standard simplex $\Delta$. After the identification, we shall refer to $T$ by the point $\mathbf{x}$ in $W_{r_{0}}$ that represents it.

The first split for $\mathbf{x}=\left(\lambda_{\alpha}\right)$ picks out an arrow starting at $\pi_{0}$. We denote the endpoint of the arrow by $\pi_{\mathbf{x}, 1}$ and denote the elementary matrix associated to this split by $E_{\mathbf{x}, 1}$. The new widths $\left(\lambda_{\alpha}^{(1)}\right)$ of the bands are related to the previous by

$$
\mathbf{x}=E_{\mathbf{x}, 1} \lambda^{(1)}
$$

Given a matrix $A$ with non-negative entries, we define the projectivization $J A$ 
as a map from $\Delta$ to itself by

$$
J A(\mathbf{y})=\frac{A \mathbf{y}}{|A \mathbf{y}|}
$$

where if $\mathbf{y}=\left(y_{1}, y_{2}, \cdots, y_{d}\right)$ in co-ordinates then $|\mathbf{y}|=\sum\left|y_{i}\right|$. This shall be the norm used throughout. The norm is additive on $\mathbb{R}_{\geq 0}^{d}$, i.e., for $\mathbf{y}, \mathbf{y}^{\prime}$ in $\mathbb{R}_{\geq 0}^{d}$, $\left|\mathbf{y}+\mathbf{y}^{\prime}\right|=|\mathbf{y}|+\left|\mathbf{y}^{\prime}\right|$

In this instance, $J E_{\mathbf{x}, 1}$ has the property that the inverse image of $W_{r_{0}}$ under $J E_{\mathbf{x}, 1}$ is some configuration space $W_{r_{1}} \in F$. This implies that for $\mathbf{x}^{(1)}=\lambda^{(1)} /\left|\lambda^{(1)}\right| \in W_{r_{1}}$

$$
J E_{\mathbf{x}, 1}\left(\mathbf{x}^{(1)}\right)=\mathbf{x}
$$

The same operation as above can be now applied to the point $\mathbf{x}^{(1)}$. Iterating in this manner, one gets a directed path in $G$ with vertices $\pi_{\mathbf{x}, n}$, and for each split $\pi_{\mathbf{x}, n-1} \rightarrow \pi_{\mathbf{x}, n}$ the associated elementary matrix $E_{\mathbf{x}, n}$. In addition, we get a sequence of configuration spaces $W_{r_{n}}$ and points $\mathbf{x}^{(n)} \in W_{r_{n}}$, constructed iteratively, such that they satisfy $\mathbf{x}^{(n-1)}=J E_{\mathbf{x}, n} \mathbf{x}^{(n)}$. Let

$$
Q_{\mathbf{x}, n}=E_{\mathbf{x}, 1} E_{\mathbf{x}, 2} \ldots E_{\mathbf{x}, n}
$$

The actual (or un-normalized) widths $\lambda^{(n)}$ of the bands at each stage $\pi_{\mathbf{x}, n}$ satisfy $\mathbf{x}=Q_{\mathbf{x}, n} \lambda^{(n)}$ and hence are in the projective class of $\mathbf{x}^{(n)}$. The sets $J Q_{\mathbf{x}, n}\left(W_{r_{n}}\right)$ form a nested sequence in $W_{r_{0}}$, all containing $\mathbf{x}$. We thus have an expansion for $\mathbf{x}$, in which each stage of the expansion is encoded by a determinant 1 projective linear map with non-negative entries.

Now we show that, almost surely, one gets an infinite expansion. Recall that the set for which a split is not defined is given by $\lambda_{\alpha_{0}}=\lambda_{\alpha_{1}}$, where $\alpha_{0}$ and $\alpha_{1}$ are the bands in the critical positions. This is always a codimension 1 
condition in $\Delta$; hence for classical i.e.m. it is measure zero. The set of classical i.e.m. for which the splitting sequence stops in a finite number of steps is a countable union of such measure zero sets and hence measure zero.

For non-classical interval exchanges, $\lambda_{\alpha_{0}}=\lambda_{\alpha_{1}}$ is a codimension 1 condition in the configuration space, except when the $\alpha_{0}$ and $\alpha_{1}$ are the only orientationreversing bands in the interval exchange. In this case, we simply amalgamate together the ends of $\alpha_{0}$ and $\alpha_{1}$ in the critical positions into a single band and cut that part of the band out of the interval $I$. This makes it measure equivalent to a classical i.e.m. with $(d-1)$ bands. So almost surely iterated splitting gives an infinite expansion.

An infinite expansion traces out an infinite directed path in the reduced diagram $G$. As mentioned before, connected components of $G$ need not be strongly connected. However each connected component has at least one strongly connected piece, i.e., any vertex in this piece splits down to any other vertex in the piece and moreover no vertex in the piece can split to something outside. Dunfield and Thurston, D. [9] call this a sink. In theory, a connected component of $G$ can have more than one sink. More generally, for linear involutions, Boissy and Lanneau [6] give a precise criteria under which a linear involution splits down to end up in some sink. The vertices that are not in any sink are called transient vertices. They are transient in the sense that if $\pi_{0}$ is not in any sink, then a generic expansion has some initial finite portion consisting of transient vertices after which it ends up in some sink. Once it is in a sink, all the vertices from then on are non-transient. In particular, depending on the initial sequence of transients, different expansions may end up in different sinks. For our purposes, these subtleties do not matter because the issue of uniform distortion in almost every expansion makes no reference to which sink we are in. So to simplify matters, we shall assume that $\pi_{0}$ belongs 
to some sink of $G$ to avoid transient vertices completely.

A finite directed path $\pi_{0} \rightarrow \pi_{1} \rightarrow \ldots \rightarrow \pi_{n}$ in $G$ shall be called a stage in the expansion. There are elementary matrices $E_{i}$ associated to each split $\pi_{i-1} \rightarrow \pi_{i}$. Let

$$
Q_{n}=E_{1} E_{2} \ldots E_{n}
$$

The image of $J Q_{n}$ in $W_{r_{0}}$ is the set of all $\mathbf{x} \in W_{r_{0}}$ whose expansion begins with this finite sequence, i.e., $\pi_{\mathbf{x}, i}=\pi_{i}$ for all $i \leq n$.

Let $W_{r_{n}}=\left(J Q_{n}\right)^{-1} W_{r_{0}}$. Subsequently, we will be interested in estimating the Lebesgue measure of subsets of $J Q_{n}\left(W_{r_{n}}\right)$. Here, by Lebesgue measure we mean the probability measure on $W_{r_{0}}$ given by the $(d-2)$-volume form induced on it as a sub-manifold of $\Delta$, normalized so that the total volume of $W_{r_{0}}$ is 1 .

For example, to get an estimate of $\ell\left(J Q_{n}\left(W_{r_{n}}\right)\right)$, we first push-forward, by $J Q_{n}$, the volume form on $W_{r_{n}}$. Since there are finitely many configurations spaces, the volumes of any two configuration spaces $W_{r}$ and $W_{s}$ differ up to some factor that depends only on $d$. So now compare the actual measure on $W_{r_{0}}$ to the push-forward. The Radon-Nikodym derivative of the actual measure with respect to the push-forward is just the Jacobian of $J Q_{n}$, restricted as a map from $W_{r_{n}}$ to $W_{r_{0}}$. So integrating the Jacobian over $W_{r_{n}}$ gives us $\ell\left(J Q_{n}\left(W_{r_{n}}\right)\right)$ up to the factor that relates the volumes of the two configuration spaces. This shows that to give quantitative estimates, one needs to understand the Jacobian of $J Q_{n}$ restricted as a map from $W_{r_{n}}$ to $W_{r_{0}}$. We denote this Jacobian by $\mathcal{J}\left(J Q_{n}\right)$.

Suppose a stage $\pi_{n}$ is combinatorially the same as $\pi_{0}$ and suppose $\Gamma$ is a finite splitting sequence starting from $\pi_{0}$. If the Jacobian $\mathcal{J}\left(J Q_{n}\right)$ is roughly the same at all points, then the relative probability that $\Gamma$ follows $\pi_{n}$ is also roughly the same as the probability that an expansion starts with $\Gamma$. We make 
the notion of the Jacobian being roughly the same at all points, precise below.

Definition 4.3.1. Suppose $\pi_{0} \rightarrow \pi_{1} \rightarrow \ldots \pi_{n}$ is a finite directed path in the reduced diagram $G$ and $Q_{n}$ the associated matrix. For $C>1$, we say that the stage $\pi_{n}$ is $C$-uniformly distorted if for all $\mathbf{y}, \mathbf{y}^{\prime} \in W_{r_{n}}$

$$
\frac{1}{C} \leq \frac{\partial\left(J Q_{n}\right)(\mathbf{y})}{\partial\left(J Q_{n}\right)\left(\mathbf{y}^{\prime}\right)} \leq C
$$

At this point, we adopt some conventions: At any stage, points in $W_{r_{0}}$, or more generally in its ambient simplex shall be denoted by x's, points in $W_{r_{n}}$, or more generally in its ambient simplex, by y's, points in $Q_{n} W_{r_{n}}$, or more generally in the image under $Q_{n}$ of the ambient simplex, by w's. In all cases, we use suitable subscripts whenever necessary. From the previous discussion $\mathbf{y}=\mathbf{x}^{(n)}$, where $\mathbf{x}^{(n)}$ is the $n$-th point constructed iteratively in the expansion of $\mathbf{x}$. The main technical theorem is

Theorem 4.3.2. Suppose $\pi_{0} \rightarrow \pi_{1} \rightarrow \ldots \rightarrow \pi_{n}$ is a stage in the expansion. There exists a constant $C>1$, independent of the stage, such that for almost every $\mathbf{x} \in J Q_{n}\left(W_{r_{n}}\right)$, there is some $m>n$, depending on $\mathbf{x}$, such that the stage $\pi_{\mathbf{x}, m}$ is $C$-uniformly distorted. 


\section{Chapter 5}

\section{Kerckhoff's Approach: Some Examples}

We fix some notation. The initial labeling at $\pi_{0}$ induces a labeling at any later stage. So it makes sense to talk of the band with label $\alpha$ at any stage. Let $Q$ be the matrix associated to a later stage. Then the column of $Q$ corresponding to $\alpha$ shall be denoted by $Q(\alpha)$.

Before we present examples, we note that for classical i.e.m. Equation (6.0.1) implies that $C$-uniform distortion is equivalent to the $C^{1 / d}$-distribution of the columns of the associated matrix, i.e., the ratio of the norms of any two columns of the matrix must be in the interval $\left(1 / C^{1 / d}, C^{1 / d}\right)$.

\subsection{Classical interval exchange with two bands}

As the first example consider a classical i.e.m. with two bands. There is just one combinatorial type of such an interval exchange and hence the Rauzy diagram has just a single vertex. At the starting stage, we shall label the band in the critical position on the bottom as 1 and the other band as 2. At every stage, we normalize so that the sum of the widths of the bands is one. If at

any stage, band 1 splits band 2 , then we denote the split by the symbol $L$ and if it happens the other way round, then we denote it by $R$. The matrices 
corresponding to $L$ and $R$ which we also denote by the same letters are:

$$
L=\left[\begin{array}{ll}
1 & 1 \\
0 & 1
\end{array}\right], \quad R=\left[\begin{array}{ll}
1 & 0 \\
1 & 1
\end{array}\right]
$$

Suppose $Q$ is the matrix at some stage in the expansion and suppose that $|Q(2)|>|Q(1)|$. Following this stage, as long as band 2 keeps splitting band 1, the first column keeps moving the second column. Hence the new matrix has the property

$$
Q^{\prime}(2)=Q(2)+k Q(1), \quad Q^{\prime}(1)=Q(1)
$$

where $k$ is the number of times band 2 has split band 1 . So the norm of the second column keeps increasing while the first column stays the same, making the inequality $|Q(2)|>|Q(1)|$ more and more pronounced. But then, as soon as band 1 splits band 2, the second column moves the first. So using additivity of the norm, we get the inequality

$$
\frac{1}{2}<\frac{\left|Q^{\prime}(1)\right|}{\left|Q^{\prime}(2)\right|}<2
$$

Thus the columns become 2-distributed. To summarize, as long as a sequence of $L$ 's occur, the columns get farther and farther from being nicely distributed. The resulting stage is farther and farther from being uniformly distorted. But as soon as a $R$ occurs after that, the columns get 2-distributed, resulting in a stage that is uniformly distorted.

Now using the measure theory of projective linear maps from a 1-simplex to itself, specifically Equation (7.2.5), it can be shown that the probability of the second column increasing in norm by a factor of $K$ due to a sequence of 
$L$ 's, is bounded away from 1 by a quantity that depends only on $K$ and is independent of the stage. This means that with a definite probability, some $R$ must occur, giving us 2-distribution and an instance of the theorem.

\subsection{The general approach}

To handle classical i.e.m.'s with $d$ bands, Kerckhoff [13] first proves a similar proposition about increase in norm: At any stage in the expansion, the probability that a band is never split, before the norm of its column increases by a large enough factor $K$, is bounded away from 1 . The bound depends only on $K$ and $d$ and is independent of the stage. In fact, the bound monotonically goes to zero as $K$ goes to infinity. As in the example above, the proof of this proposition uses Equation (7.2.5).

After this, Kerckhoff proves the following inductive step: Suppose our stage has a collection of $C^{\prime}$-distributed columns that also includes the column with the largest norm. Then there is a definite probability that one gets a larger collection of $C^{\prime \prime}$-distributed columns that includes the largest column at that instant, $C^{\prime \prime}$ depending only on $C^{\prime}$ and $d$ and independent of the stage. Iterating the inductive step shows that with a definite probability, one must get $C$ distributed.

The basic idea behind the inductive step is: Before the norms of the

columns in the collection increase by a factor of $K$, suppose one of the following happens: either an outside column becomes the column with the largest norm or a column in the collection moves a column from outside. At this point, if we add the outside band to our collection, then similar to the example above, the ratio of the norms of any two columns in it is within suitable bounds, even if initially, the ratio is way out. So it remains to show that with a definite 
probability, one of the two events happens.

The key idea is that this happens provided there are no isolated blocks. An isolated block is a splitting sequence in which there is a collection of bands that satisfy the properties: First, every band in the collection is moved, at least once, by some other band in the collection and second, every band in the collection moves some other band in the collection but never moves a band outside the collection. Kerckhoff shows that for irreducible classical i.e.m., isolated blocks are absent.

The proposition about increase in norm implies that with a definite probability, every band in the collection has to move some other band before its norm increases by $K$. But it could so happen that it moves a band in the collection itself. If this repeats enough number of times, then Kerckhoff shows that there is a sub-collection that forms an isolated block. The number of times it needs to repeat is independent of the stage. Finally, since isolated blocks are absent, there is a definite probability that a band in the collection has to move a band outside, which is exactly the kind of split we want to finish the proof of the inductive step.

\subsection{Issues with non-classical interval exchanges}

With non-classical interval exchanges, there are two issues: First, the probabilities of splits are different from the ratios of the measures of the ambient simplices. This phenomena makes the precise statement of the proposition about increase in norm incorrect in this context. Second, isolated blocks are possible. To illustrate the first issue, consider the splitting sequence in Figure 5.3.1. 




Figure 5.3.1: Splitting sequence

The matrix $Q$ associated to the stage is

$$
Q=\left[\begin{array}{cccc}
1 & 0 & 0 & 0 \\
0 & n+1 & n+2 & 0 \\
2 & n & n+1 & 0 \\
0 & 0 & 0 & 1
\end{array}\right]
$$

Denote the simplex with the columns of $Q$ as vertices by $\Delta(Q)$. Let the image under $Q$, of the configuration space for the stage be $W(Q)$.

As in section 4 , let $e_{i j}$ be the midpoint of the edge $\left[e_{i}, e_{j}\right]$. As shown in Figure 5.3.2, in the standard simplex $\Delta$, the initial configuration space $W$ is the quadrilateral with vertices $e_{13}, e_{14}, e_{24}$ and $e_{23}$.

Let $Q(i j)$ denote the midpoint of the edge $[Q(i), Q(j)]$ of $\Delta(Q)$. As shown in Figure 5.3.3, $W(Q)$ is the quadrilateral in $\Delta(Q)$ with vertices $Q(12), Q(13), Q(34)$ and $Q(24)$. 


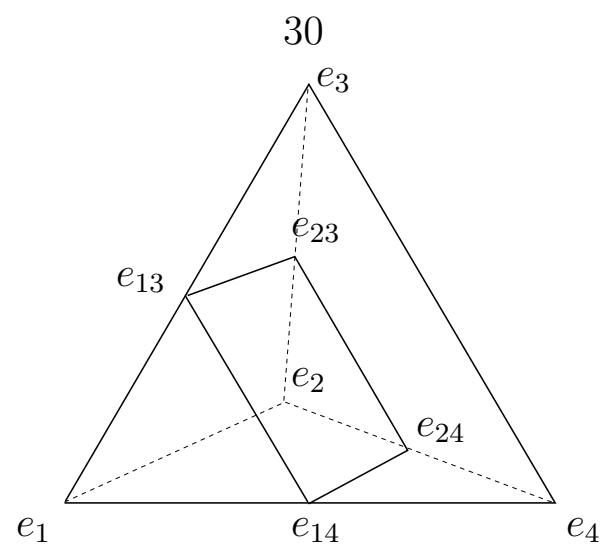

Figure 5.3.2: $W$ in $\Delta$

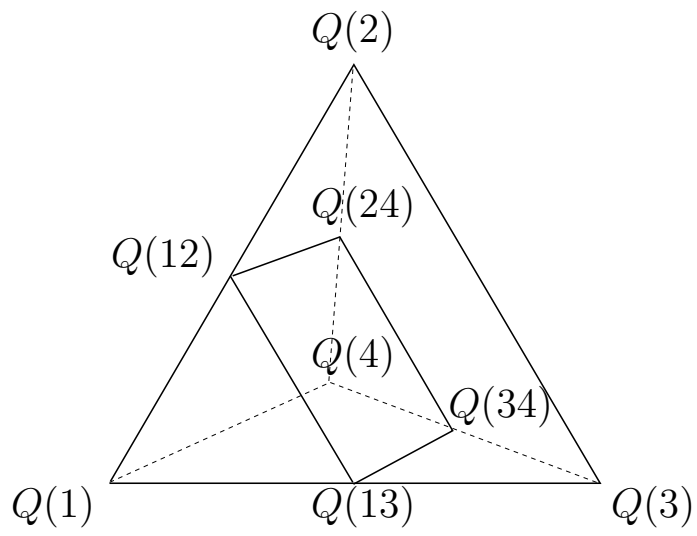

Figure 5.3.3: $W(Q)$ in $\Delta(Q)$

The columns representing the vertices of $W(Q)$ are

$$
\begin{aligned}
& Q(12)=\left[\begin{array}{c}
1 / 2 \\
(n+1) / 2 \\
(n+2) / 2 \\
0
\end{array}\right], Q(13)=\left[\begin{array}{c}
1 / 2 \\
(n+2) / 2 \\
(n+3) / 2 \\
0
\end{array}\right], Q(24)=\left[\begin{array}{c}
(n+1) / 2 \\
n / 2 \\
0 \\
(n+2) / 2 \\
1 / 2
\end{array}\right] \\
& {\left[\begin{array}{c}
(n+1) / 2 \\
1 / 2
\end{array}\right],}
\end{aligned}
$$

Projectivize to find the images of these vertices in the configuration space $W$ 
of the starting stage

$$
\begin{aligned}
& J Q(12)=\left[\begin{array}{c}
1 / 2(n+2) \\
(n+1) / 2(n+2) \\
1 / 2 \\
0
\end{array}\right], J Q(13)=\left[\begin{array}{c}
1 / 2(n+3) \\
(n+2) / 2(n+3) \\
1 / 2 \\
0
\end{array}\right] \\
& J Q(34)=\left[\begin{array}{c}
0 \\
1 / 2 \\
(n+1) / 2(n+2) \\
1 / 2(n+2)
\end{array}\right], J Q(24)=\left[\begin{array}{c}
0 \\
1 / 2 \\
n / 2(n+1) \\
1 / 2(n+1)
\end{array}\right]
\end{aligned}
$$

In terms of the vertices of the quadrilateral $W$, the linear combinations are



Figure 5.3.4: Projection of W(Q) 
By symmetry, the quadrilateral $W$ is a square with side length $1 / \sqrt{2}$. $W(Q)$ projects down as shown in the Figure 5.3.4. From this we calculate the area of the quadrilateral $J Q(12) J Q(24) J Q(34) J Q(13)$ to be $1 / 2(n+1)(n+2)(n+3)$.

Now imagine that in the next split band 1 splits band 2. As shown in Figure 5.3.5, the split is represented by the smaller simplex with vertices $Q(2), Q(3), Q(4)$ and $Q(12)$. The part of $W(Q)$ that lies inside this smaller simplex is the part of $W(Q)$ that lies above the shaded plane in Figure 5.3.5. This is the triangle $Q(12) Q(24) Q(34)$. Projecting the triangle down to $W$ gives the triangle $J Q(12) J Q(24) J Q(34)$ in Figure 5.3.4. The area of this triangle is $1 / 4(n+1)(n+2)^{2}$.

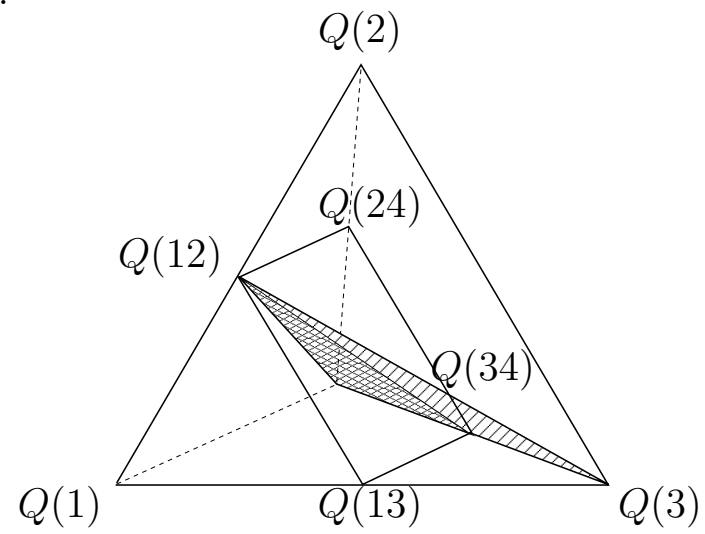

Figure 5.3.5: Band 1 splits Band 2

Hence the probability that band 1 splits band 2 is the ratio of the area of triangle with vertices $J Q(12) J Q(24) J Q(34)$ to the area of quadrilateral with vertices $J Q(12) J Q(24) J Q(34) J Q(13)$ viz. $(n+3) / 2(n+2)$, which is close to $1 / 2$ for large $n$. On the other hand, when band 1 splits band 2, $Q(2)$ moves $Q(1)$. As a result, $|Q(1)|$ increases by a factor of $2(n+2) / 3$. In particular, by choosing a stage for $n$ large enough, one can arrange that the probability of the split is close to $1 / 2$ even though the norm increase of $Q(1)$ is by a factor as large as we want. It is interesting to compare this to the ratio $\ell(J(Q E)(\Delta)) / \ell(J Q(\Delta))$ where $E$ is the elementary matrix for band 1 splitting 
band 2. By Lemma 7.1.1, the ratio is equal to the reciprocal of the factor by which $|Q(1)|$ increases, i.e., $3 / 2(n+2)$. This is completely different from the probability of the split.

The example given above shows that for non-classical interval exchanges, the statement of the proposition about increase in norm cannot be true as it stands. However, a closer look reveals that for the proof of the inductive step to work, the proposition about increase in norm needs to hold for the largest columns around. In Proposition 8.0.5, we show that this is indeed true for non-classical interval exchanges.

The second issue is that for non-classical interval exchanges, isolated blocks arise whenever there is a combinatorial reduction. However, irreducibility of a non-classical interval exchange implies that one splits out of these blocks with a definite probability. See Step 1 of Proposition 8.0.21.

From the next section on, we begin the technical details starting with the analysis of the Jacobian of the restriction of the projective linear map to the configuration spaces. 


\section{Chapter 6}

\section{Jacobian of the Restriction}

Let $\pi_{0} \rightarrow \pi_{1} \rightarrow \cdots \rightarrow \pi_{n}$ be a stage. For the rest of the paper, we simplify the notation introduced in Chapter 4 by dropping the subscripts. Henceforth we will denote $\pi_{n}$ by $\pi$, the configuration spaces $W_{r_{n}}$ and $W_{r_{0}}$ at $\pi_{n}$ and $\pi_{0}$ by $W$ and $W_{0}$ and the associated matrix $Q_{n}$ by $Q$. In this section, we analyze the Jacobian of $J Q$ restricted as a map from $W$ to $W_{0}$. The expression for the Jacobian of full map $J Q$ from $\Delta$ to itself is known [7]. At a point in the configuration space $W$, we write down a matrix for the derivative of the full map $J Q$ with respect to suitable decompositions as direct sums, of the tangent spaces to $\Delta$ at the point itself and its image under $J Q$. Then using the expression for the full Jacobian and the particular form of this matrix, we get an expression for the Jacobian of $J Q$ as a map from $W$ to $W_{0}$.

We fix some terminology. Given an affine subspace $L$ of $\mathbb{R}^{d}$ and some $\mathbf{y} \in \mathbb{R}^{d}$ which need not be in $L$, we let $T_{\mathbf{y}} L$ be the subspace of the tangent space at $\mathbf{y} \in \mathbb{R}^{d}$ parallel to $L$. We will denote a vector in $\mathbb{R}^{d}$ and also in the tangent space at any point $\mathbf{y}$, by the same letter $\mathbf{u}$, whenever the context is clear. Associated to a co-dimension 1 subspace $L$ transverse to a vector $\mathbf{u}$, there is a projection map $\phi_{\mathbf{u}}: T_{\mathbf{y}} \mathbb{R}^{d} \rightarrow T_{\mathbf{y}} L$ by projecting along lines parallel to $\mathbf{u}$ till one hits $L$. A dilation of $\mathbb{R}^{d}$ by $t>0$ shall be denoted by $\rho_{t}$. The derivative of a linear map $A$ acting on tangent spaces is denoted by $A$ itself. 
Let $\Delta(Q)$ be the simplex with vertices the columns of $Q$. The map $J Q$ : $\Delta \rightarrow \Delta$ is a diffeomorphism onto its image. This means that, for any point $\mathbf{y} \in$ $\Delta$, the vector $\mathbf{w}=Q \mathbf{y}$ is transverse to both $\Delta(Q)$ and $\Delta$. Hence at the level of tangent spaces $\mathbf{w}$ is transverse to $T_{\mathbf{w}} \Delta(Q)$ and $T_{\mathbf{w}} \Delta$. So the projection map $\phi_{\mathbf{w}}: T_{\mathbf{w}} \mathbb{R}^{d} \rightarrow T_{\mathbf{w}} \Delta$ by projecting along lines parallel to $\mathbf{w}$ is an isomorphism from $T_{\mathbf{w}} \Delta(Q)$ to $T_{\mathbf{w}} \Delta$. The full derivative $\mathbf{D} J Q(\mathbf{y}): T_{\mathbf{y}} \Delta \rightarrow T_{J Q(\mathbf{y})} \Delta$ is the composition

$$
T_{\mathbf{y}} \Delta \stackrel{Q}{\rightarrow} T_{\mathbf{w}} \Delta(Q) \stackrel{\phi_{\mathbf{w}}}{\longrightarrow} T_{\mathbf{w}} \Delta \stackrel{\rho_{|\mathbf{w}|^{-1}}}{\longrightarrow} T_{J Q(\mathbf{y})} \Delta
$$

It is known that (see Bufetov [7]) the Jacobian $\mathcal{J}_{\Delta}(J Q)$ of the above composition is given by

$$
\mathcal{J}_{\Delta}(J Q)(\mathbf{y})=\frac{1}{|\mathbf{w}|^{d}}=\frac{1}{|Q \mathbf{y}|^{d}}
$$

Fix unit vectors $\mathbf{m}_{0}$ and $\mathbf{m}$ normal to $W_{0}$ and $W$ respectively such that the tangent bundle $T \Delta$ has the orthogonal decompositions

$$
T \Delta=\left(\mathbb{R} \mathbf{m}_{0}\right) \oplus T W_{0} \quad, \quad T \Delta=(\mathbb{R} \mathbf{m}) \oplus T W
$$

where $T W_{0}$ and $T W$ are the tangent bundles of the configuration spaces.

Since $J Q$ restricted to $W$ maps it into $W_{0}$ and is a diffeomorphism onto its image, at any point $\mathbf{y} \in W$ the full derivative $\mathbf{D} J Q$ restricts to an isomorphism from $T_{\mathbf{y}} W$ to $T_{J Q(\mathbf{y})} W_{0}$. So with respect to the orthogonal decompositions fixed above, the matrix at $\mathbf{y}$ for $\mathbf{D} J Q$ has the form:

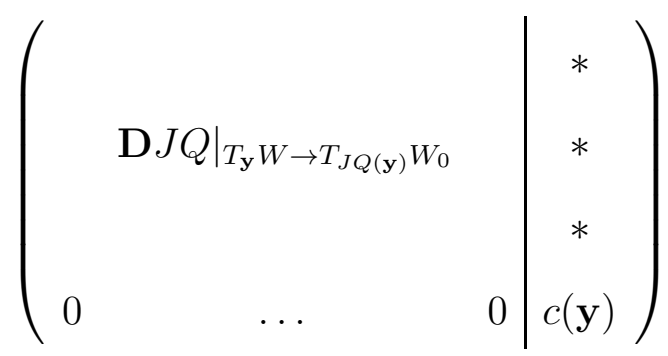


From the matrix form we get

$$
\mathcal{J}_{\Delta}(J Q)(\mathbf{y})=c(\mathbf{y}) \mathcal{J}(J Q)(\mathbf{y})
$$

To compute $c(\mathbf{y})$, we use the fact that

$$
\mathbf{D} J Q(\mathbf{y})=\rho_{|\mathbf{w}|^{-1}} \circ \phi_{\mathbf{w}} \circ Q
$$

In the composition, we project the vector $Q \mathbf{m} \in T_{\mathbf{w}} \Delta(Q)$ by lines parallel to $\mathbf{w}$ to get $\phi_{\mathbf{w}}(Q \mathbf{m}) \in T_{\mathbf{w}} \Delta$. Next we write $\phi_{\mathbf{w}}(Q \mathbf{m})$ as the unique linear combination

$$
\phi_{\mathbf{w}}(Q \mathbf{m})=a(\mathbf{y}) \mathbf{m}_{0}+\mathbf{p}
$$

in $T_{\mathbf{w}} \Delta$, where $\mathbf{p} \in T_{\mathbf{w}} W_{0}$. Last we apply the dilation $\rho_{|\mathbf{w}|^{-1}}$ to get $c(\mathbf{y})=$ $a(\mathbf{y}) /|\mathbf{w}|$. Hence from equation (6.0.2) we get

$$
\mathcal{J}(J Q)(\mathbf{y})=\frac{1}{a(\mathbf{y})|\mathbf{w}|^{d-1}}=\frac{1}{a(\mathbf{y})|Q \mathbf{y}|^{d-1}}
$$

Lemma 6.0.4. As $\mathbf{y}$ varies over $W$, the quantity a $(\mathbf{y})$ remains constant.

Proof. To prove the lemma, we need to understand the projection $\phi_{\mathbf{w}}: T_{\mathbf{w}} \Delta(Q) \rightarrow$ $T_{\mathbf{w}} \Delta$. We refer to Figure 6.0.5 which is a schematic picture of $T_{\mathbf{w}} \mathbb{R}^{d}$. In the figure, the projection $\phi_{\mathbf{w}}$ by lines parallel to $\mathbf{w}$ is represented by the horizontal dashed arrows. Let $L$ be the co-dimension 1 subspace in $T_{\mathbf{w}} \mathbb{R}^{d}$ spanned by $\mathbf{w}$ and $T_{\mathbf{w}} W_{0}$. If $A_{0}$ denotes the subspace of $\mathbb{R}^{d}$ spanned by $W_{0}$, i.e., the subspace of $\mathbb{R}^{d}$ satisfying the equation that defines $W_{0}$, then $L=T_{\mathbf{w}} A_{0}$. So $L$ does not depend on $\mathbf{w}$.

Since $\phi_{\mathbf{w}}$ gives an isomorphism from $T_{\mathbf{w}} W(Q)$ to $T_{\mathbf{w}} W_{0}, T_{\mathbf{w}} W(Q)$ is in $L$. In the figure, we represent $L$ by the horizontal $(x, y)$-plane. The vector $\mathbf{m}_{0}$ is 




Figure 6.0.5: Schematic picture

orthogonal to $T_{\mathbf{w}} W_{0}$ and transverse to $\mathbf{w}$, so in fact transverse to $L$.

For the remainder of the proof, we shall denote the $(d-1)$-dimensional subspace of $\mathbb{R}^{d}$ that is parallel to $\Delta$ by $\Delta$ itself. The essential point from the discussion above is: thinking of each tangent space $T_{\mathbf{w}} \mathbb{R}^{d}$ as $\mathbb{R}^{d}$ itself, there are two $(d-1)$-dimensional transverse subspaces $L$ and $\Delta$ with a vector $\mathbf{m}_{0} \in \Delta$ not in $L$. Moreover, as $\mathbf{y}$ varies over $W$ which is equivalent to saying as $\mathbf{w}$ varies over $W(Q)$, the vector $\mathbf{w}$ is constrained to lie in $L$. Any vector $\mathbf{v} \in T_{\mathbf{w}} \mathbb{R}^{d}$ can be uniquely written as

$$
\mathbf{v}=a \mathbf{m}_{0}+\mathbf{q}
$$

where $\mathbf{q} \in L$. Applying the projection $\phi_{\mathbf{w}}: T_{\mathbf{w}} \mathbb{R}^{d} \rightarrow T_{\mathbf{w}} \Delta$

$$
\phi_{\mathbf{w}}(\mathbf{v})=a \phi_{\mathbf{w}}\left(\mathbf{m}_{0}\right)+\phi_{\mathbf{w}}(\mathbf{q})=a \mathbf{m}_{0}+\phi_{\mathbf{w}}(\mathbf{q})
$$

The projection $\phi_{\mathbf{w}}$ has the form $\phi_{\mathbf{w}}(\mathbf{v})=\mathbf{v}-b \mathbf{w}$. This implies that the vector $\phi_{\mathbf{w}}(\mathbf{q})$ belongs to $L$. So the component of $\phi_{\mathbf{w}}(\mathbf{v})$ along $\mathbf{m}_{0}$ remains $a \mathbf{m}_{0}$, even 
as $\mathbf{w}$ varies. Choosing $\mathbf{v}=Q \mathbf{m}$ we are done.

By the above lemma, we can drop the dependence of $a(\mathbf{y})$ on $\mathbf{y}$ in the expression for the Jacobian and write it just as $a$. However the number $a$ does depend on the stage of the expansion. Observe that, up to the number $a$, the expression for the Jacobian of the restriction looks similar to the Jacobian of a determinant 1 projective linear map with non-negative entries in dimension $(d-1)$. We shall make this observation precise and use it to compute measures in Chapter 8.

Next, we give the definition for a set of $C$-distributed vectors.

Definition 6.0.6. Let $C>1$. A set of vectors $\left\{\mathbf{u}_{1}, \mathbf{u}_{2}, \ldots, \mathbf{u}_{k}\right\}$ are said to be $C$-distributed if

$$
\frac{1}{C}<\frac{\left|\mathbf{u}_{i}\right|}{\left|\mathbf{u}_{j}\right|}<C
$$

for all $i, j$ such that $1 \leq i, j \leq k$.

We conclude from the previous lemma that a stage $\pi$ is $C$-uniformly distorted if and only if the vertices of $W(Q)$ are $C^{\frac{1}{d-1}}$-distributed. Moreover if the vertices of $\Delta(Q)$ are $C$-distributed, then the vertices of $W(Q)$ are also $C$-distributed. So to show that for almost every $\mathbf{x}$, there exists a stage $\pi_{\mathbf{x}, n}$ that is $C$-uniformly distorted, it is enough to show that for almost every $\mathbf{x}$, there exists a stage $\pi_{\mathbf{x}, n}$ such that the columns of $Q_{\mathbf{x}, n}$ are $C$-distributed. The proof of this roughly follows Kerckhoff's original proof in the case of classical i.e.m.'s while weakening various hypothesis.

In the next section, we recall the measure theory for determinant 1 projective linear maps with non-negative entries, from the standard simplex into itself. Additionally, we evaluate measures of certain subsets of the standard simplex, which shall be useful later. 


\section{Chapter 7}

\section{Measure Theory}

\subsection{Measure theory of projective linear maps}

Let $Q: \mathbb{R}^{d} \rightarrow \mathbb{R}^{d}$ be a linear map with non-negative entries and determinant 1. Projectivize $Q$ to get the map $J Q: \Delta \rightarrow \Delta$. We recall 4.4 Lemma 1 from Yoccoz [20]:

Lemma 7.1.1.

$$
\frac{\ell(J Q(\Delta))}{\ell(\Delta)}=\frac{1}{\prod_{\alpha=1}^{d}|Q(\alpha)|}
$$

Remark 7.1.2. The formula for $\ell(J Q(\Delta))$ can be derived by integrating the expression 6.0.1 for $\mathcal{J}_{\Delta}(J Q)$ on $\Delta$.

The next corollary does not need the assumption that the matrix $Q$ has determinant 1. This will be relevant in Step 5 of Proposition 8.0.5.

Corollary 7.1.3. Let $E$ be an elementary matrix with the off-diagonal $(\beta, \alpha)$ entry equal to $R>0$ and let $Q$ be a linear map with non-negative entries. Then

$$
\frac{\ell(J(Q E)(\Delta))}{\ell(J Q(\Delta))}=\frac{|Q(\alpha)|}{|Q(\alpha)|+R|Q(\beta)|}
$$

Proof. As both sides of the claimed equality are unchanged if we replace $Q$ by 
$t Q$, we can reduce to the case $\operatorname{det}(Q)=1$. By applying Lemma 7.1.1, we get

$$
\frac{\ell(J(Q E)(\Delta))}{\ell(J Q(\Delta))}=\frac{\prod_{\gamma=1}^{d}|Q(\gamma)|}{\prod_{\gamma=1}^{d}|Q E(\gamma)|}=\frac{|Q(\alpha)|}{|Q E(\alpha)|}=\frac{|Q(\alpha)|}{|Q(\alpha)|+R|Q(\beta)|}
$$

\subsection{Evaluating measures}

We apply Corollary 7.1.3 to evaluate measures of certain subsets of $\Delta$ that we shall encounter in Chapter 9.

\subsubsection{Wedges}

For a pair of distinct labels $\alpha, \beta \in S$ and a non-negative constant $R$, let $\Delta_{R}(\alpha, \beta)$ be the set of points in $\Delta$ whose convex combination $\mathbf{x}=\sum \lambda_{\gamma} e_{\gamma}$ satisfies

$$
\frac{\lambda_{\beta}}{\lambda_{\alpha}} \geq R
$$

Figure 7.2.3 shows a schematic picture of $\Delta_{R}(\alpha, \beta)$.

The shaded plane $F_{R}(\alpha, \beta)$ represents the set of points for which $\lambda_{\beta} / \lambda_{\alpha}=R$. The set $\Delta_{R}(\alpha, \beta)$ is the region in Figure 7.2.3 that lies below the shaded plane. Thus it looks like a wedge in $\Delta$.

Let $Q: \mathbb{R}^{d} \rightarrow \mathbb{R}^{d}$ be a linear map with nonnegative entries. Write $R$ in the form

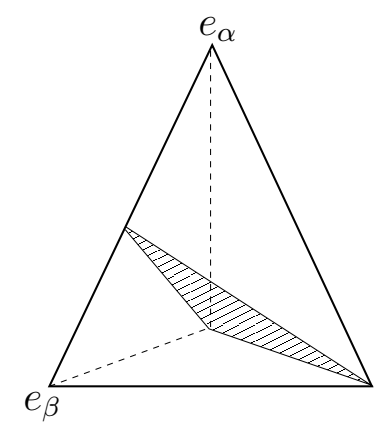

Figure 7.2.3: $\Delta_{R}(\alpha, \beta)$

$$
R=K \frac{|Q(\alpha)|}{|Q(\beta)|}
$$


for some $K>0$. Then

$$
J Q\left(\Delta_{R}(\alpha, \beta)\right)=J(Q E)(\Delta)
$$

where $E$ is the elementary matrix with the $(\beta, \alpha)$ entry equal to $R$. Applying Corollary 7.1.3, we get

$$
\frac{\ell\left(J Q\left(\Delta_{R}(\alpha, \beta)\right)\right)}{\ell(J Q(\Delta))}=\frac{1}{1+K}
$$

\subsubsection{Half-space intersections}

Having established Equation (7.2.5) for the relative measure of wedges, we move on to more general subsets of $\Delta$ which we call thin half-space intersections. The wedges $\Delta_{R}(\alpha, \beta)$ are an instance of these. We encounter thin half-space intersections in the proof of Proposition 8.0.5.

A half-space $H$ in $\mathbb{R}^{d}$ that intersects $\Delta$ and does not contain the vertex $e_{\alpha}$ shall be called a half-space opposite $\alpha$. Let $S_{H}$ denote the set of those $\gamma \neq \alpha$ for which the intersection $\partial H \cap\left[e_{\alpha}, e_{\gamma}\right]$ is non-empty, where $\left[e_{\alpha}, e_{\gamma}\right]$ denotes the edge of $\Delta$ joining $e_{\alpha}$ with $e_{\gamma}$. For $R_{0}>0$, a half-space $H$ opposite $\alpha$ is said to be $R_{0}$-thin if for every $\gamma \in S_{H}$, the intersection point $a_{\alpha \gamma}=\partial H \cap\left[e_{\alpha}, e_{\gamma}\right]$ has a convex combination that satisfies

$$
\frac{\lambda_{\gamma}}{\lambda_{\alpha}} \geq R_{0}
$$

We have the following inclusion

Lemma 7.2.7. For $R=R_{0} / d$, we have

$$
H \cap \Delta \subset \bigcup_{\gamma \in S_{H}} \Delta_{R}(\alpha, \gamma)
$$


Proof. Let $F$ be the sub-simplex of $\Delta$ whose vertices are indexed by $S_{H} \cup\{\alpha\}$, and let $F\left(R_{0}\right)$ denote the set of points in $F$ whose convex combination in $F$ satisfies $\lambda_{\alpha} \leq 1 /\left(1+R_{0}\right)$. Let $\Delta\left(R_{0}\right)$ be the convex hull of $F\left(R_{0}\right)$ with the remaining vertices of $\Delta$.

\section{Claim 7.2.8.}

$$
H \cap \Delta \subset \Delta\left(R_{0}\right)
$$

Proof. Since both $H \cap \Delta$ and $\Delta\left(R_{0}\right)$ are convex sets, it is enough to show that every vertex of $H \cap \Delta$ is in $\Delta\left(R_{0}\right)$. By the virtue of being $R_{0}$-thin, the vertices $a_{\alpha \gamma}$ are in $\Delta\left(R_{0}\right)$. All the other vertices of $H \cap \Delta$ lie in $F(\alpha)$, the face of $\Delta$ opposite $e_{\alpha}$, which itself lies in $\Delta\left(R_{0}\right)$.

The lemma then follows from the claim

\section{Claim 7.2.9.}

$$
\Delta\left(R_{0}\right) \subset \bigcup_{\gamma \in S_{H}} \Delta_{R}(\alpha, \gamma)
$$

Proof. Notice that the face $F(\alpha)$ of $\Delta$ is in the union above. The vertices of $\Delta\left(R_{0}\right)$ that are not in $F$ lie in $F_{\alpha}$. So it is enough to show that $F\left(R_{0}\right)$ lies in the union. The convex combination of a point in $F\left(R_{0}\right)$ satisfies $\lambda_{\alpha} \leq 1 /\left(1+R_{0}\right)$. This implies that for at least one $\gamma \in S_{H}$,

$$
\lambda_{\gamma} \geq\left(\frac{1}{\# S_{H}}\right)\left(1-\frac{1}{1+R_{0}}\right)>\frac{R_{0}}{d\left(1+R_{0}\right)}
$$

It follows that

$$
\frac{\lambda_{\gamma}}{\lambda_{\alpha}}>\frac{R_{0}}{d}
$$

and so the point belongs to $\Delta_{R}(\alpha, \gamma)$.

This finishes the proof of Lemma 7.2.7. 
In Proposition 8.0.5, we need an upper bound on the measure of half-space intersections. This upper bound is derived there by using the inclusion of Lemma 7.2.7 and then bounding the measures of the individual wedges in the union.

We now have all the preliminaries to carry out the proof of Theorem 4.3.2. 


\section{Chapter 8}

\section{Proof of Theorem 4.3.2}

Let $\pi$ be a stage in the expansion. Let $W$ be the configuration space at $\pi$ and let $Q$ be the matrix associated to $\pi$.

Proposition 8.0.1. For almost every $\mathbf{x} \in J Q(W)$, every $\alpha \in S$ is split infinitely often and splits other bands infinitely often.

Proof. The first claim is that

Claim 8.0.2. Suppose in the expansion for some $\mathbf{x}$, a band $\alpha \in S$ occurs in the critical positions infinitely often. Then $\alpha$ is split infinitely often and splits other bands infinitely often.

Proof. Suppose $\alpha$ is split finitely many times. Then, there exists a future stage $\pi_{\mathbf{x}, m}$ after which $\alpha$ never gets split. So the actual width of $\alpha$ remains unchanged from $\pi_{\mathbf{x}, m}$ onwards. On the other hand, after $\pi_{\mathbf{x}, m}$, the band $\alpha$ splits some other band $\gamma$ infinitely often; this is impossible if the actual width of $\alpha$ is to remain fixed.

Alternatively, suppose $\alpha$ splits other bands finitely many times. A band leaves a critical position only if it splits some other band. Since $\alpha$ gets split infinitely often, there exists a future stage $\pi_{\mathbf{x}, m}$ after which $\alpha$ remains fixed in one of the critical positions and is the only band split thereafter. But then from $\pi_{\mathbf{x}, m}$ onwards, the actual widths of the rest of the bands remain 
unchanged because they never get split. This makes it impossible to split $\alpha$ ad infinitum.

Thus, to prove Proposition 8.0.1, it is enough to show that almost surely, every band $\alpha$ occurs in the critical positions infinitely often. Let $Z$ be the set of those $\mathbf{x}$ in $J Q(W)$ for which there exists a band $\alpha$ that occurs in the critical positions finitely often in the expansion of $\mathbf{x}$. Fix such a point $\mathbf{x}$ and let $S_{1}$ be the subset of those bands that occur in the critical positions finitely many times in the expansion of $\mathbf{x}$. Then there is a future stage $\pi_{\mathbf{x}, n}$ such that in the expansion of $\mathbf{x}$ after $\pi_{\mathbf{x}, n}$

1. The bands in $S_{1}$ never occur in the critical positions.

2. Every band in $S_{2}=S \backslash S_{1}$ occur in the critical positions infinitely often.

Moreover we assume that $\pi_{\mathbf{x}, n}$ is the first instance in the expansion of $\mathbf{x}$ in which the above properties hold.

Claim 8.0.3. $S=S_{1} \sqcup S_{2}$ is a combinatorial reduction of $\pi_{\mathbf{x}, n}$ and all future stages.

Proof. Without loss of generality, suppose some $\beta \in S_{2}$ occurs to the left of some $\alpha \in S_{1}$ on the bottom. Since $\alpha$ never enters a critical position, whenever $\beta$ is split, the split has to begin at the other end of $\beta$. This increases the number of bands $k(\alpha)$ to the left of $\alpha$ on the bottom by 1 . Moreover since $\beta$ is split infinitely often, the number $k(\alpha)$ has to become large enough to force $\alpha$ into the critical position on the bottom which is a contradiction. This proves the claim.

Let $H$ be the set of finite splitting sequences $\jmath$ starting from $\pi$ such that final stage of $\jmath$ is combinatorially reducible. The set $H$ is countable because it is a subset of the countable set of finite splitting sequences. 
By the above claim, we can define a map $\psi: Z \rightarrow H$. Let $Z_{\jmath}=\psi^{-1}(\jmath)$. If the set $Z_{\jmath}$ is non-empty, then the expansion after $\pi$ of each point in $Z_{\jmath}$ begins with the sequence $\jmath$. Since $H$ is a countable set, $\ell(Z)=\sum_{\jmath \in H} \ell\left(Z_{\jmath}\right)$. So to prove Proposition 8.0.1 it is enough to show that $\ell\left(Z_{\jmath}\right)=0$ for all $\jmath$ in $H$.

Claim 8.0.4. For every $\jmath$ in $H, \ell\left(Z_{\jmath}\right)=0$.

Proof. Let $S=S_{1} \sqcup S_{2}$ be the combinatorial reduction for the final stage $\pi^{\prime}$ of $\jmath$. Let $\left(\lambda_{\alpha}^{\prime}\right)$ denote the normalized widths at stage $\pi^{\prime}$. We will show that a point in $Z_{\jmath}$ must satisfy constraint $(4.2 .3)$ in the widths $\left(\lambda_{\alpha}^{\prime}\right)$. The set given by this constraint has measure zero, which then proves the claim.

For a point $\mathbf{x}$ that does not satisfy the constraint, set

$$
D=\sum_{\alpha \in S_{1, b}} \lambda_{\alpha}^{\prime}-\sum_{\beta \in S_{1, t}} \lambda_{\beta}^{\prime}
$$

and without loss of generality, assume that $D>0$. We claim that for a point with $D>0$, there is a stage in the expansion after $\pi^{\prime}$ in which twice the sum of the actual widths of the bands in $S_{2}$ is equal to $D$. When this happens, a band in $S_{1}$ is forced into the critical position on the bottom. This would show that such a point cannot be in $Z_{\jmath}$.

Let $\lambda_{\alpha}^{\infty}$ denote the limit of the actual widths of $\alpha$ in the expansion of $\mathbf{x}$. To show that there is a stage in the expansion of $\mathbf{x}$ after $\pi^{\prime}$ in which the sum of the actual widths of the bands in $S_{2}$ is equal to $D$, we show that $\lambda_{\alpha}^{\infty}=0$ for all $\alpha \in S_{2}$. We prove this by showing that for any $\epsilon>0$, there exists a future stage at which the actual widths of all bands in $S_{2}$ are at most $\epsilon$.

For $\epsilon>0$, there exists a stage $\pi_{\mathbf{x}, N}$ after $\pi^{\prime}$ such that the actual widths $\lambda_{\alpha}^{(N)}$ at $\pi_{\mathbf{x}, N}$ satisfy $\lambda_{\alpha}^{\infty}<\lambda_{\alpha}^{(N)}<\lambda_{\alpha}^{\infty}+\epsilon$ for all $\alpha$ in $S_{2}$. Then for every subsequent split after $\pi_{\mathbf{x}, N}$, the actual width of the band split can be reduced by at most $\epsilon$. This means that the actual width of a band doing the splitting is at most 
$\epsilon$. For the bands in $S_{2}$, there is a future stage $\pi_{\mathbf{x}, N^{\prime}}$ such that every band in $S_{2}$ has split other bands at least once after $\pi_{\mathbf{x}, N}$ but before $\pi_{\mathbf{x}, N^{\prime}}$. By the previous observation, the actual widths at $\pi_{\mathbf{x}, N^{\prime}}$ of all bands in $S_{2}$ are at most $\epsilon$ and we are done.

This concludes the proof of Proposition 8.0.1.

For $\alpha \in S$ and $\mathbf{x} \in J Q(W)$, let $n(\mathbf{x}, \alpha)$ be the index of the first stage after $\pi$ such that, in the split $\pi_{n(\mathbf{x}, \alpha)} \rightarrow \pi_{n(\mathbf{x}, \alpha)+1}$ the band split is $\alpha$; if there is no such integer, set $n(\mathbf{x}, \alpha)=\infty$. From Proposition 8.0.1, we see that $n(\mathbf{x}, \alpha)$ is finite almost surely.

For $M>1$ let $X_{M, \alpha}=\left\{\mathbf{x} \in J Q(W):\left|Q_{n(\mathbf{x}, \alpha)}(\alpha)\right|>M|Q(\alpha)|\right\}$. The set $X_{M, \alpha}$ is thus the set of those $\mathbf{x}$ in whose expansion the $\alpha$-column increases in norm by a factor greater than $M$ before $\alpha$ is split.

Proposition 8.0.5. Let $\alpha$ be a band and $C>1$ be a constant such that

$$
|Q(\alpha)|>\frac{1}{C} \max _{\gamma \in S}|Q(\gamma)|
$$

Then there exists a constant $M>1$, depending only on $C$ and $d$, such that

$$
\ell\left(X_{M, \alpha}\right)<\frac{1}{2 d} \ell(J Q(W))
$$

In fact, for all $M^{\prime}>M$, the proportion of $X_{M^{\prime}, \alpha}$ in $J Q(W)$ has an upper bound that depends only on $M^{\prime}$; moreover the bound $\rightarrow 0$ as $M^{\prime} \rightarrow \infty$

Proof. We shall prove Proposition 8.0.5 in a number of steps:

Step 1:

As a first step, we shall include the set $X_{M, \alpha}$ in a finite union of sets whose measures are easier to estimate. 
Let $Y_{M, \alpha}=(J Q)^{-1}\left(X_{M, \alpha}\right)$. For a $\beta \in S$ and a positive constant $R$, recall from Chapter 7 that $\Delta_{R}(\alpha, \beta)$ is the set of points in $\Delta$ whose convex combination satisfies

$$
\frac{\lambda_{\beta}}{\lambda_{\alpha}} \geq R
$$

Write $R$ as

$$
R=K \frac{|Q(\alpha)|}{|Q(\beta)|}
$$

for some $K>0$ and let $Y_{R}(\alpha, \beta)=\Delta_{R}(\alpha, \beta) \cap W$ and let $X_{R}(\alpha, \beta)=$ $J Q\left(Y_{R}(\alpha, \beta)\right)$. We shall show that

Lemma 8.0.6. For $K=(M-1) /(d-1)$ we have the inclusion

$$
Y_{M, \alpha} \subseteq \bigcup_{\beta \neq \alpha} Y_{R}(\alpha, \beta)
$$

or equivalently

$$
X_{M, \alpha} \subseteq \bigcup_{\beta \neq \alpha} X_{R}(\alpha, \beta)
$$

Proof. Since $\alpha$ does not get split till $\pi_{n(\mathbf{x}, \alpha)}$, the column $Q_{n(\mathbf{x}, \alpha)}(\alpha)$ has the form

$$
Q_{n(\mathbf{x}, \alpha)}(\alpha)=Q(\alpha)+\sum_{\beta \neq \alpha} c_{\beta} Q(\beta)
$$

for non-negative integers $c_{\beta}$.

Claim 8.0.7. Let $\mathbf{y} \in W$ be the pre-image of $\mathbf{x}$ under JQ. The convex combination $\mathbf{y}=\sum \lambda_{\gamma} e_{\gamma}$ must satisfy

$$
\frac{\lambda_{\beta}}{\lambda_{\alpha}} \geq c_{\beta}
$$

for all $\beta$.

Proof. To simplify notation, let $m=n(\mathbf{x}, \alpha)$. Let $T$ denote the matrix asso- 
ciated to the splitting sequence $\pi \rightarrow \cdots \rightarrow \pi_{m}$. Starting with the normalized widths $\left(\lambda_{\gamma}\right), \gamma \in S$ at $\pi$, let $\left(\lambda_{\gamma}^{(m)}\right)$ denote the actual widths at $\pi_{m}$ resulting from the splitting sequence. Since $\alpha$ is never split till $\pi_{m}$, the width of $\alpha$ remains unchanged, i.e., $\lambda_{\alpha}^{(m)}=\lambda_{\alpha}$. Additionally, the entries of $\alpha$-th column of $T$ are given by $T_{\beta \alpha}=c_{\beta}$ for $\beta \neq \alpha$ and $T_{\alpha \alpha}=1$. From Chapter 4 , the relationship between the old and the new widths is

$$
\lambda_{\beta}=\sum_{\gamma \in S} T_{\beta \gamma} \lambda_{\gamma}^{(m)}
$$

Since all terms on the right hand side above are non-negative, we get the inequality

$$
\lambda_{\beta} \geq T_{\beta \alpha} \lambda_{\gamma}^{(m)}=c_{\beta} \lambda_{\alpha}
$$

finishing the proof of the claim.

Claim 8.0.8. If $\left|Q_{n(\mathbf{x}, \alpha)}(\alpha)\right| \geq M|Q(\alpha)|$ for some constant $M>1$, then for at least one $\beta$

$$
c_{\beta} \geq \frac{M-1}{d-1} \frac{|Q(\alpha)|}{|Q(\beta)|}
$$

Proof. By the additive property of the norm on $\left(\mathbb{R}_{\geq 0}\right)^{d}$

$$
\left|Q_{n(\mathbf{x}, \alpha)}(\alpha)\right|=|Q(\alpha)|+\sum_{\beta \neq \alpha} c_{\beta}|Q(\beta)|
$$

If $\left|Q_{n(\mathbf{x}, \alpha)}(\alpha)\right| \geq M|Q(\alpha)|$, then

$$
\sum_{\beta \neq \alpha} c_{\beta}|Q(\beta)| \geq(M-1)|Q(\alpha)|
$$

which implies the claim.

By Claims 8.0.7 and 8.0.8, for each $\mathbf{y} \in Y_{M, \alpha}$, there is some $\beta$ such that the 
convex combination for $\mathbf{y}$ satisfies

$$
\frac{\lambda_{\beta}}{\lambda_{\alpha}} \geq K \frac{|Q(\alpha)|}{|Q(\beta)|}
$$

for $K=(M-1) /(d-1)$. This proves the lemma.

To show that there is a choice of $M$ large enough such that $\ell\left(X_{M, \alpha}\right)$ is bounded above by $(1 / 2 d) \ell(J Q(W))$, it is enough to show that there is a $M$ large enough such that for every $\beta, \ell\left(J Q\left(Y_{R}(\alpha, \beta)\right)\right)$ is bounded above by $\left(1 / 2 d^{2}\right) \ell(J Q(W))$. So for the remainder of this proof, we focus on one such $Y_{R}(\alpha, \beta)$.

Step 2:

Figure 8.0.9 shows for some $\beta$, a schematic picture of the sets $\Delta_{R}(\alpha, \beta)$ and $Y_{R}(\alpha, \beta)$ inside $\Delta$. The simplex is drawn such that the vertex $e_{\alpha}$ is on the top and the opposite face $F(\alpha)$ is in the horizontal plane forming the floor of the simplex.

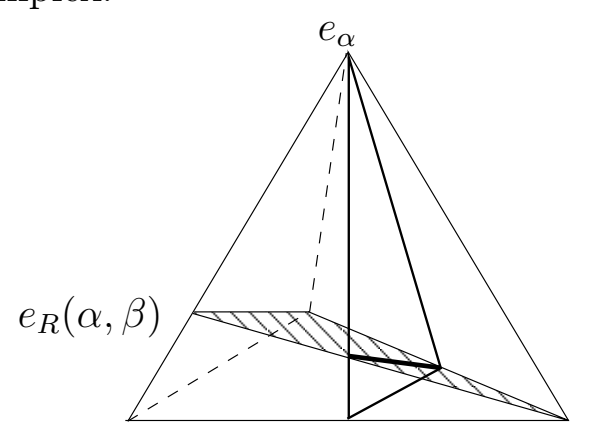

$e_{\beta}$

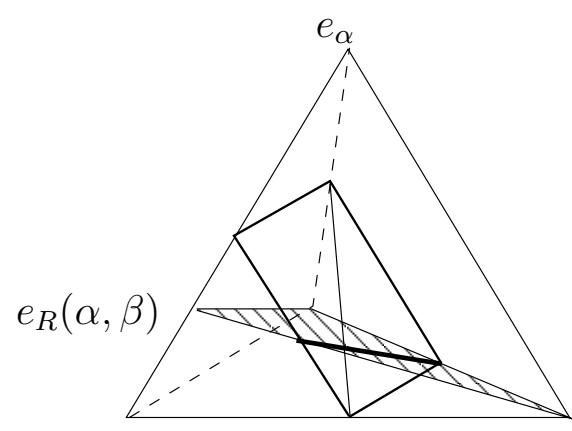

$e_{\beta}$

Figure 8.0.9: Schematic Picture

The first picture is an instance when $\alpha$ is orientation preserving and the second picture is an instance when $\alpha$ is orientation-reversing. Let $e_{R}(\alpha, \beta)$ be the point on the edge $\left[e_{\alpha}, e_{\beta}\right]$ whose convex combination satisfies $\lambda_{\beta} / \lambda_{\alpha}=R$. Let $F_{R}(\alpha, \beta)$ denote the convex hull of $e_{R}(\alpha, \beta)$ and the vertices of the simplex 
other than $e_{\alpha}$ and $e_{\beta}$. In the picture, it is represented by the plane that is shaded. Then $\Delta_{R}(\alpha, \beta)$ is the wedge in the simplex bounded by $F_{R}(\alpha, \beta)$ on the top and $F(\alpha)$ on the bottom, i.e., it is the region in the simplex below the shaded plane. As a result, $Y_{R}(\alpha, \beta)$ is the part of $W$ that lies below the intersection of the shaded plane and $W$, which is shown by the bold line segment in either picture.

The position of $e_{R}(\alpha, \beta)$ on the edge joining $e_{\alpha}$ and $e_{\beta}$ is determined by the value of $K$ and the ratio $|Q(\alpha)| /|Q(\beta)|$. The assumption $|Q(\alpha)|>$ $(1 / C) \max _{\gamma \in S}|Q(\gamma)|$ implies that the ratio $|Q(\alpha)| /|Q(\beta)|$ is bounded below by $1 / C$. Using this lower bound, we see that the points in $\Delta_{R}(\alpha, \beta)$ and hence in $Y_{R}(\alpha, \beta)$ satisfy

$$
\frac{\lambda_{\beta}}{\lambda_{\alpha}} \geq \frac{K}{C}
$$

This means that, for every $\beta$, the distance along the edge of the point $e_{R}(\alpha, \beta)$ from $e_{\beta}$ is bounded above by a quantity that depends only on $M$ and $C$. Moreover, this quantity goes to 0 as $M$ goes to infinity. In other words, each wedge is uniformly thin.

Step 3:

In this step, we triangulate $W$ by $(d-2)$-dimensional simplices $\Delta(i)$ in a specific way. Then to get the upper bound for measure relative to $J Q(W)$, it is enough to get the same bound for measure of the intersection with each simplex in the triangulation.

Recall from Chapter 4, each configuration space $W$ is a convex hull of a finite number of vertices, either of type $e_{\alpha \beta}$ or $e_{\gamma}$. Fix a triangulation of $W$ by $(d-2)$-dimensional simplices as follows:

1. Triangulate $\partial W$ without introducing new vertices. This can be done in 
any manner by adding an appropriate number of new diagonals.

2. Fix the vertex $p$ of $W$ where if $\alpha$ is orientation preserving then $p=e_{\alpha}$, and if $\alpha$ is orientation-reversing then $p=e_{\alpha \gamma}$ for some $\gamma$. Cone off the triangulation of $\partial W$ to $p$ to get a triangulation of $W$.

For example, in the second figure of Figure 8.0.9, we have triangulated by adding in the diagonal of the quadrilateral. The number of simplices $\Delta(i)$ in a triangulation is bounded above by some number $n(d)$ that depends only on $d$.

Let the individual simplices in the triangulation of $W$ be $\Delta(i)$. By construction, each $\Delta(i)$ contains the vertex $p$ of $W$. To show the required bound for $\ell\left(X_{R}(\alpha, \beta)\right)$, it is enough to show that for $M$ large enough, the ratio $\ell\left(J Q\left(Y_{R}(\alpha, \beta) \cap \Delta(i)\right)\right) / \ell(J Q(\Delta(i)))$ is bounded above by $1 / 2 d^{2}$ for each $i$. Taking union over all $i$ implies the required bound for $X_{R}(\alpha, \beta)$. So for the remainder of this proof, we will focus on one such $\Delta(i)$.

Step 4:

The intersection $Y_{R}(\alpha, \beta) \cap \Delta(i)$ is a half-space intersection $H \cap \Delta(i)$ with $\partial H=F_{R}(\alpha, \beta)$ (see Chapter 7). In Inequality (8.0.10), if $K / C>1 / 2$, then the half-space intersection is opposite $p$. In this step, we shall show that $H \cap \Delta(i)$ opposite $p$ is $R / 2$-thin. Hence we can use Lemma 7.2.7 to include the half-space in a union of wedges in $\Delta(i)$. This reduces the task to showing a suitable upper bound for the ratio in each of these wedges.

The edges of $\Delta(i)$ with endpoint $p$ that have non-empty intersection with $F_{R}(\alpha, \beta)$ are the ones joining $p$ to a vertex $q$ of $\Delta(i)$ in $F_{\alpha}$. Denote the set of vertices of $\Delta(i)$ in $F_{\alpha}$ by $S_{H}$ and for each vertex $q \in S_{H}$, let $a_{q}$ denote the point of intersection $F_{R}(\alpha, \beta) \cap[p, q]$. Since $a_{q} \in F_{R}(\alpha, \beta)$, it's convex combination 
in the full simplex $\Delta$ satisfies

$$
\frac{\lambda_{\beta}}{\lambda_{\alpha}}=R
$$

Let $a_{q}$ have the convex combination $a_{q}=\lambda_{p} p+\lambda_{q} q$ in $\Delta(i)$. Write

$$
p=\frac{e_{\alpha}+e_{\gamma}}{2}, q=\frac{e_{\beta}+e_{\eta}}{2}
$$

where $\gamma=\alpha$ or $\gamma \neq \alpha$ depending on whether $\alpha$ is orientation preserving or orientation-reversing, and similarly $\eta=\beta$ or $\eta \neq \beta$. This means that $\lambda_{\alpha}=\lambda_{p}$ or $\lambda_{\alpha}=\lambda_{p} / 2$, and similarly $\lambda_{\beta}=\lambda_{q}$ or $\lambda_{\beta}=\lambda_{q} / 2$. In any case, combining this with Equation (8.0.11) implies that convex combinations in $\Delta(i)$ of the points on the line segment joining $a_{q}$ to $q$ satisfy

$$
\frac{\lambda_{q}}{\lambda_{p}} \geq \frac{R}{2}
$$

Thus our half-space intersection with $\Delta(i)$ opposite $p$ is $R / 2$-thin. By Lemma 7.2.7, for $R_{0}=R / 2(d-1)$, we have the inclusion

$$
Y_{R}(\alpha, \beta) \cap \Delta(i) \subset \bigcup_{q \in S_{H}} \Delta(i)_{R_{0}}(p, q)
$$

Using Equation (8.0.12), the ratio $|Q p| /|Q q|$ satisfies

$$
\frac{|Q p|}{|Q q|}=\frac{|Q(\alpha)|+|Q(\gamma)|}{|Q(\beta)|+|Q(\eta)|}<(C+1) \frac{|Q(\alpha)|}{|Q(\beta)|}
$$

So if we set

$$
R_{1}=\left(\frac{K}{4 d C}\right) \frac{|Q p|}{|Q q|}
$$


then $R_{0}>R_{1}$. This implies the inclusion

$$
\Delta(i)_{R_{0}}(p, q) \subset \Delta(i)_{R_{1}}(p, q)
$$

for each $q \in S_{H}$. Combining it with inclusion (8.0.13), we get

$$
Y_{R}(\alpha, \beta) \cap \Delta(i) \subset \bigcup_{q \in S_{H}} \Delta(i)_{R_{1}}(p, q)
$$

The number of simplices $\Delta(i)$ is at most $n(d)$. So to establish the required upper bound on $\ell\left(J Q\left(Y_{R}(\alpha, \beta) \cap \Delta(i)\right)\right)$, it is enough to show that there is a choice of $M$ and consequently of $R_{1}$ such that

$$
\ell\left(J Q\left(\Delta(i)_{R_{1}}(p, q)\right)\right) \leq \frac{1}{2 d^{2} n(d)} \ell(J Q(\Delta(i)))
$$

Step 5:

Equation (7.2.5) applies to projective linear maps with non-negative entries of the standard $(d-1)$-dimensional simplex into itself. So to apply Equation (7.2.5) to get Estimate (8.0.14), we need to extract such a map $J \widehat{Q}$ : $\Delta_{d-2} \rightarrow \Delta_{d-2}$ from the restriction of $J Q$ to $\Delta(i)$. This creates two issues: First, to extract the map $J \widehat{Q}$, we have to identify $\Delta(i)$ with $\Delta_{d-2}$; this introduces a distortion in the standard measure on $\Delta_{d-2}$. So we need to show that this distortion is universally bounded. Second, we need to show that the Jacobian $\mathcal{J}(J Q)$ is, up to a universal constant, the same as the full Jacobian $\mathcal{J}_{\Delta_{d-2}}(J \widehat{Q})$ of the extracted map. In the final step, we show how to do this.

Recall from Chapter 4 that the volume forms on configuration spaces are normalized so that the volume of each configuration space is 1 . Hence, up to the ratios of the configuration space volumes, for a measurable subset $X$ of 
$J Q(W)$

$$
\ell(X) \approx \int_{W} \mathcal{J}(J Q)(\mathbf{y}) \chi_{Y}(\mathbf{y}) d \mathbf{y}
$$

where $\chi_{Y}$ is the characteristic function of the set $Y=(J Q)^{-1}(X)$. Using (8.0.15), we get

$$
\frac{\ell\left(J Q\left(\Delta(i)_{R_{1}}(p, q)\right)\right)}{\ell(J Q(\Delta(i)))}=\frac{\int_{\Delta(i)_{R_{1}}(p, q)} \mathcal{J}(J Q)(\mathbf{y}) d \mathbf{y}}{\int_{\Delta(i)} \mathcal{J}(J Q)(\mathbf{y}) d \mathbf{y}}
$$

Recall from Chapter 6, the expression for the Jacobian of the restriction to $W$.

$$
\mathcal{J}(J Q)(\mathbf{y})=\frac{1}{a|Q \mathbf{y}|^{d-1}}
$$

So

$$
\frac{\ell\left(J Q\left(\Delta(i)_{R_{1}}(p, q)\right)\right)}{\ell(J Q(\Delta(i)))}=\frac{\int_{\Delta(i)_{R_{1}}(p, q)} \frac{1}{|Q \mathbf{y}|^{d-1}} d \mathbf{y}}{\int_{\Delta(i)} \frac{1}{|Q \mathbf{y}|^{d-1}} d \mathbf{y}}
$$

In Equation (8.0.16), the integrand looks similar to the Jacobian of a determinant 1 projective linear map, with non-negative entries, of a standard $(d-2)$-dimensional simplex $\Delta_{d-2}$. We indicate how to make this observation precise and then use it to bound the ratio from above.

Let $A_{0}$ be the subspace in $\mathbb{R}^{d}$ spanned by $W_{0}$ and let $A$ be the subspace in $\mathbb{R}^{d}$ spanned by $W$. Thus $A_{0}$ and $A$ are subspaces of $\mathbb{R}^{d}$ satisfying the equations that define $W_{0}$ and $W$ respectively. Fix some linear isomorphism $F: \mathbb{R}^{d-1} \rightarrow$ $A_{0}$ such that $F\left(\Delta_{d-2}\right)$ contains $W_{0}$. Also, up to a permutation of the standard basis in $\mathbb{R}^{d-1}$, there is a unique linear isomorphism $G(i): \mathbb{R}^{d-1} \rightarrow A$ such that $G(i)\left(\Delta_{d-2}\right)=\Delta(i)$. Use the identification $G(i)$ to label the vertices of $\Delta_{d-2}$ by the corresponding labels of $\Delta(i)$.

Using the map $F$ and the identifications $G(i)$, we define a linear map 
$\widehat{Q}=F^{-1} \circ Q \circ G(i)$. Since the projectivization $J \widehat{Q}$ maps $\Delta_{d-2}$ into itself, the map $\widehat{Q}$ has non-negative entries.

Since there are finitely many configuration spaces and finitely many simplices in the triangulation of each configuration space, the distortion by $G(i)$ of the standard Lebesgue measure on $\Delta_{d-2}$ is bounded above by a constant that depends only on $d$. This means that for a point $\mathbf{y} \in \Delta_{d-2}$, the quantity $1 /|Q G(i) \mathbf{y}|^{d-1}$ (the integrand on the right in (8.0.16)) is the same as the full Jacobian $J_{\Delta_{d-2}}(J \widehat{Q})(\mathbf{y})=1 /|\widehat{Q} \mathbf{y}|^{d-1}$, up to a constant that depends only on $d$. This also means that up to a universal constant, the norm of the $r$-th column $|\widehat{Q}(r)|$ is the same as $|Q r|$.

The restriction $\Delta(i)_{R_{1}}(p, q) \rightarrow J Q\left(\Delta(i)_{R_{1}}(p, q)\right)$ corresponds to $J(\widehat{Q} E):$ $\Delta_{d-2} \rightarrow \Delta_{d-2}$ where $E$ is the elementary matrix whose off-diagonal entry in the $(q, p)$ place is $R_{1}$.

Applying Corollary 7.1.3, we get

$$
\begin{aligned}
\frac{\ell\left(J Q\left(\Delta(i)_{R_{1}}(p, q)\right)\right)}{\ell(J Q(\Delta(i)))} & \approx \frac{|\widehat{Q}(p)|}{|\widehat{Q}(p)|+R_{1}|\widehat{Q}(q)|} \\
& =|\widehat{Q}(p)|\left[|\widehat{Q}(p)|+\left(\frac{K}{4 d C}\right) \frac{|Q p||\widehat{Q}(q)|}{|Q q|}\right]^{-1} \\
& \approx \frac{4 d C}{4 d C+K}<\frac{4 d C}{K}
\end{aligned}
$$

Finally, if $M$ is large enough such that $K>8 d^{3} n(d) C$, then we get Estimate (8.0.14); moreover it is easily checked that as $M \rightarrow \infty, K \rightarrow \infty$ and so the second part of the Proposition 8.0.5 also follows.

In the next lemma, we use the technique in Step 5 of Proposition 8.0.5 to estimate from below, the probability of certain splits. This shall be useful later in Proposition 8.0.21. 
Lemma 8.0.17. Let $\gamma$ and $\sigma$ be the bands in critical positions at some stage $\pi_{1}$. Let $C>1$ be a constant such that

$$
|Q(\gamma)|>\frac{1}{C} \max _{\alpha \in S}|Q(\alpha)|
$$

Then up to a universal constant depending only on $d$, the probability that $\gamma$ splits $\sigma$ is bounded below by the quantity $1 / n(d)(2 C)^{d}$.

Proof. Let $\pi_{2}$ be the stage resulting from $\gamma$ splitting $\sigma$. Let $W_{1}$ and $W_{2}$ denote the configuration spaces corresponding to $\pi_{1}$ and $\pi_{2}$.

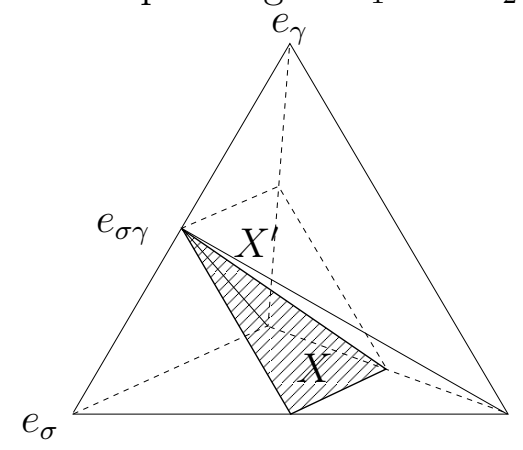

Figure 8.0.18: $\gamma$ splits $\sigma$

A schematic picture is shown in Figure 8.0.18 with the shaded part $X$ in $W_{1}$ representing the split. In other words, the shaded part $X$ is the image of $W_{2}$ under the projectivization of the elementary matrix associated to the split. Similarly, let $X^{\prime}$ be $W_{1} \backslash X$; this corresponds to $\sigma$ splitting $\gamma$ instead. Except when $\gamma$ is the only orientation-reversing band on its side, $X^{\prime}$ is non-empty. When $X^{\prime}$ is empty, the split occurs with probability 1 ; so we focus on the split in which $X^{\prime}$ is non-empty. Let $Z$ denote $\partial X \cap \partial X^{\prime}$, i.e., the part of the boundary that separates the two sets.

The vertices of $W_{1}$ fall into the following categories:

1. If a vertex has the linear combination $(1 / 2) e_{\sigma}+(1 / 2) e_{\tau}$ with $\tau \neq \gamma$, then it lies in $X \backslash Z$. 
2. If a vertex has the linear combination $(1 / 2) e_{\gamma}+(1 / 2) e_{\tau}$ with $\tau \neq \sigma$, then it lies in $X^{\prime} \backslash Z$.

3. All other vertices of $W_{1}$ lie in $Z$.

Just as in Proposition 8.0.5, let $\Delta^{\prime}(i)$ be the triangulation of $X^{\prime}$. Denote the set of vertices of $\Delta^{\prime}(i)$ by $V^{\prime}(i)$. Let $i_{0}$ be the index for which the measure $\ell\left(J Q_{1}\left(\Delta^{\prime}\left(i_{0}\right)\right)\right)$ is the maximum among all $i$. The triangulation has at most $n(d)$ simplices. Hence we get the estimate

$$
\ell\left(J Q_{1}\left(X^{\prime}\right)\right) \leq n(d) \ell\left(J Q_{1}\left(\Delta^{\prime}\left(i_{0}\right)\right)\right)
$$

Let $V_{Z}$ be the subset of vertices of $\Delta^{\prime}\left(i_{0}\right)$ that lie in $Z$. Construct any triangulation of $X$ such that there is a simplex $\Delta(j)$ for which the points in $V_{Z}$ are vertices. Then

$$
\frac{\ell\left(J Q_{1}(X)\right)}{\ell\left(J Q_{1}\left(X^{\prime}\right)\right)}>\left(\frac{1}{n(d)}\right) \frac{\ell\left(J Q_{1}(\Delta(j))\right)}{\ell\left(J Q_{1}\left(\Delta^{\prime}\left(i_{0}\right)\right)\right)}
$$

So it is enough to show a lower bound for the right side in the equation above. Let $V(j)$ denote the set of vertices of $\Delta(j)$. Associated to the simplices $\Delta^{\prime}\left(i_{0}\right)$ and $\Delta(j)$, we define maps $\widehat{Q_{1}}\left(i_{0}\right)=F^{-1} \circ Q_{1} \circ G^{\prime}\left(i_{0}\right)$ and $\widehat{Q_{1}}(j)=$ $F^{-1} \circ Q_{1} \circ G(j)$ from the standard $(d-2)$-dimensional simplex into itself. See Step 5 of Proposition 8.0.5 for the definition of these maps. The gist of the discussion in that step is that up to a universal constant introduced in the identifications,

$$
\frac{\ell\left(J Q_{1}(\Delta(j))\right)}{\ell\left(J Q_{1}\left(\Delta^{\prime}\left(i_{0}\right)\right)\right)} \approx \frac{\ell\left(J \widehat{Q_{1}}(j)\left(\Delta_{d-2}\right)\right)}{\ell\left(J \widehat{Q_{1}}\left(i_{0}\right)\left(\Delta_{d-2}\right)\right)}
$$

There is a unique linear isomorphism $G\left(j i_{0}\right)$ of $\mathbb{R}^{d-1}$ such that $\widehat{Q_{1}}\left(i_{0}\right)=\widehat{Q_{1}}(j) \circ$ $G\left(j i_{0}\right)$. Since the number of identification maps $G(*)$ is finite, the number of linear isomorphisms that relate them is also finite. Hence the ratio of the 
determinants of $\widehat{Q_{1}}(*)$ is universally bounded. This bound can be incorporated in applying Lemma 7.1.1 to evaluate the right hand side in (8.0.19). Recall that the norm of the $r$-th column $\widehat{Q_{1}}(*)(r)$ is up to a universal constant the same as the norm of $Q r$. In conclusion,

$$
\frac{\ell\left(J \widehat{Q_{1}}(j)\left(\Delta_{d-2}\right)\right)}{\ell\left(J \widehat{Q_{1}}\left(i_{0}\right)\left(\Delta_{d-2}\right)\right)} \approx \frac{\prod_{p \in V^{\prime}\left(i_{0}\right)}\left|Q_{1} p\right|}{\prod_{q \in V(j)}\left|Q_{1} q\right|}=\frac{\prod_{p \in V^{\prime}\left(i_{0}\right) \backslash V_{Z}}\left|Q_{1} p\right|}{\prod_{q \in V(j) \backslash V_{Z}}\left|Q_{1} q\right|}
$$

In (8.0.20), the number of vertices in the numerator is exactly equal to the number of vertices in the denominator. So the terms in the products in the numerator and the denominator pair off. In addition, any vertex $p$ in $V^{\prime}\left(i_{0}\right) \backslash V_{Z}$ is a vertex in $X^{\prime} \backslash Z$, i.e., in our categorization, the vertex belongs to category (2). Hence it's linear combination is $p=(1 / 2) e_{\gamma}+(1 / 2) e_{\tau}$, and so it satisfies $\left|Q_{1} p\right|>\left|Q_{1}(\gamma)\right| / 2$. Hence for a vertex $q$ in $V(j) \backslash V_{Z}$, we have the estimate

$$
\frac{\left|Q_{1} p\right|}{\left|Q_{1} q\right|}>\frac{\left|Q_{1}(\gamma)\right| / 2}{\max _{\alpha \in S}\left|Q_{1}(\alpha)\right|}>\frac{1}{2 C}
$$

Using the estimate in (8.0.20) gives the lower bound

$$
\frac{\ell\left(J \widehat{Q_{1}}(j)\left(\Delta_{d-2}\right)\right)}{\ell\left(J \widehat{Q_{1}}\left(i_{0}\right)\left(\Delta_{d-2}\right)\right)} \succ\left(\frac{1}{2 C}\right)^{d-1}
$$

Thus from (8.0.19) we get the lower bound for the probability of the split

$$
\frac{\ell\left(J Q_{1}(X)\right)}{\ell\left(J Q_{1}\left(W_{1}\right)\right)} \succ \frac{1}{1+n(d)(2 C)^{d-1}}>\frac{1}{n(d)(2 C)^{d}}
$$

The next proposition, which goes back to Kerckhoff [13], has the following idea: One starts off by arranging the columns $Q(\alpha)$ in the order of decreasing 
norm and then picks out a $C_{0}$-distributed subset $\mathcal{A}_{0}$ starting with the column with the largest norm and following the order. Then the proposition shows that there is a definite probability, independent of the stage, that some future stage has a larger subset $\mathcal{A} \supsetneq \mathcal{A}_{0}$ that is $C_{1}$-distributed and contains the column with the largest norm, with the constant $C_{1}$ independent of the stage. Thus the proposition can be used as an iterative step which we iterate over a finite number of times to get, with a definite probability, a future stage that is $C$-distributed. The precise statement of the proposition here is identical to 4.4 Lemma 5 in Yoccoz [20], except that it is formulated for non-classical interval exchanges.

Proposition 8.0.21. Let $C_{0}>1$ be a constant and let $\mathcal{A}_{0} \subsetneq S$ be a set of $C_{0}$-distributed columns which contains the largest column in norm. Then there exists constants $c_{1} \in(0,1)$ and $C_{1}>1$, depending only on $C_{0}$ and $d$, and a finite set of future stages $\pi_{\theta}$, after $\pi$, that satisfy the following two conditions:

1. Denote the matrix corresponding to each $\pi_{\theta}$ by $Q_{\theta}$ and the configuration space by $W_{\theta}$. The sets $J Q_{\theta}\left(W_{\theta}\right)$ have disjoint interiors and

$$
\sum_{\theta} \ell\left(J Q_{\theta}\left(W_{\theta}\right)\right) \geq c_{1} \ell(J Q(W))
$$

2. For every $\theta$, there exists a set of $C_{1}$-distributed columns $\mathcal{A}_{\theta} \supsetneq \mathcal{A}_{0}$ that contains the largest column at the stage $\pi_{\theta}$.

Proof. We recall the basic idea of the proof from Chapter 5: Before the norms of columns in $\mathcal{A}_{0}$ increase by some factor, suppose one of the following happens: either a column outside $\mathcal{A}_{0}$ becomes the column with the largest norm or a column in $\mathcal{A}_{0}$ moves a column outside. At this point, if we let $\mathcal{A}$ be $\mathcal{A}_{0}$ union this outside column, then as we shall see, the new collection is $C_{1}$-distributed 
where $C_{1}$ depends only on the initial constants. Then it remains to show that with a definite probability, one of the two events happens.

The proof here follows the proof of 4.4 Lemma 5 in Yoccoz [20] closely, except that the individual steps require justification for non-classical interval exchanges. The steps are as follows:

Step 1:

To make it possible for a column in $\mathcal{A}_{0}$ to move a column outside, a column outside $\mathcal{A}_{0}$ needs to land in one of the critical positions. In this step, we show that there is a definite probability that this happens before the maximum of the norms of the columns increases by a definite factor. Precisely, we claim that $\pi$ can be split to a future stage $\pi_{\iota}$ such that, for constants $c_{1}^{\prime} \in(0,1)$ and $C_{1}^{\prime}>1$ depending only on $C_{0}$ and $d$, we have

$$
\begin{gathered}
\max _{\alpha \in S}\left|Q_{\iota}(\alpha)\right|<C_{1}^{\prime} \max _{\alpha \in S}|Q(\alpha)| \\
\ell\left(J Q_{\iota}\left(W_{\iota}\right)\right)>c_{1}^{\prime} \ell(J Q(W))
\end{gathered}
$$

and at least one of the bands in the critical positions in $\pi_{\iota}$ does not belong to the collection $\mathcal{A}_{0}$.

Proof. Consider the $\operatorname{sink} G_{0}$ of the reduced diagram $G$ that contains $\pi_{0}$. The nodes in $G$ correspond to unlabeled combinatorial types. By Proposition 8.0.1, for each node $\pi$ in $G_{0}$ and for each band in $\pi$, there are splitting sequences which bring that band to a critical position. For each band in $\pi$, choose one such splitting sequence. The set of chosen splitting sequences over all nodes of $G_{0}$ is equal to $d$ times the number of nodes in $G_{0}$. This means that there is an upper bound $h$, depending only on $d$, on the lengths of all chosen splitting sequences. 
Now we introduce the labeling. For some band $\beta$ in $S \backslash \mathcal{A}_{0}$, let $\iota(\beta)$ be the chosen splitting sequence starting from $\pi$ that brings $\beta$ into one of the critical positions. Let $\iota$ be the shortest prefix of $\iota(\beta)$ that has a band, which is not in $\mathcal{A}_{0}$, in a critical position.

By construction, the length of $\iota$ is at most $h$. This implies that the norm of a column in $\mathcal{A}_{0}$ participating in $\iota$ can increase by a factor of at most $2^{h}$. So we get the estimate

$$
\max _{\alpha \in \mathcal{A}_{0}}\left|Q_{\iota}(\alpha)\right|<2^{h} \max _{\alpha \in S}|Q(\alpha)|
$$

Thus (8.0.22) holds with $C_{1}^{\prime}=2^{h}$.

To show the bound (8.0.23) for the measures, it is enough to show that every split in $\iota$ has relative probability bounded below by a constant that depends only on $C_{0}$ and $d$.

Suppose we are at some intermediate stage $\pi_{1}$ of $\iota$. Following $\pi_{1}$, suppose a band $\gamma$ in $\mathcal{A}_{0}$ splits a band $\sigma$ in $\mathcal{A}_{0}$ to give us the next stage $\pi_{2}$. The column $Q_{1}(\gamma)$ satisfies the estimate

$$
\left|Q_{1}(\gamma)\right|>|Q(\gamma)|>\frac{1}{C_{0}} \max _{\alpha \in S}|Q(\alpha)|>\frac{1}{C_{0} C_{1}^{\prime}} \max _{\alpha \in S}\left|Q_{1}(\alpha)\right|
$$

By Lemma 8.0.17, the probability of this split is bounded below by $1 / n(d)\left(2 C_{0} C_{1}^{\prime}\right)^{d}$, up to a universal constant that depends only on $d$. Finally, using the fact that the length of $\iota$ is bounded above by $h$, we get

$$
\ell\left(J Q_{\iota}\left(W_{\iota}\right)\right) \succ \frac{1}{n(d)^{h}\left(2 C_{0} C_{1}^{\prime}\right)^{d h}} \ell(J Q(W))
$$

So setting

$$
c_{1}^{\prime} \approx \frac{1}{n(d)^{h}\left(2 C_{0} C_{1}^{\prime}\right)^{d h}}
$$

proves Step 1. 
Step 2:

Notice that for every $\alpha \in \mathcal{A}_{0}$, we have $\left|Q_{\iota}(\alpha)\right| \geq\left(1 / C_{1}^{\prime}\right) \max _{\gamma \in S}\left|Q_{\iota}(\gamma)\right|$. So we can apply Proposition 8.0.5 to the stage $\pi_{\iota}$. Let

$$
X_{M, \alpha}=\left\{\mathbf{x} \in J Q_{\iota}\left(W_{\iota}\right):\left|Q_{n(\mathbf{x}, \alpha)}(\alpha)\right|>M\left|Q_{\iota}(\alpha)\right|\right\}
$$

By Proposition 8.0.5, there exists $M>1$, depending on $C_{1}^{\prime}$ and $d$ such that $\ell\left(X_{M, \alpha}\right)<(1 / 2 d) \ell\left(J Q_{\iota}\left(W_{\iota}\right)\right)$. If we let

$$
X=J Q_{\iota}\left(W_{\iota}\right) \backslash \bigcup_{\alpha \in \mathcal{A}_{0}} X_{M, \alpha}
$$

then we have the estimate

$$
\frac{\ell(X)}{\ell\left(J Q_{\iota}\left(W_{\iota}\right)\right)} \geq 1-\sum_{\alpha \in \mathcal{A}_{0}} \frac{\ell\left(X_{M, \alpha}\right)}{\ell\left(J Q_{\iota}\left(W_{\iota}\right)\right)}>1-\sum_{\alpha \in \mathcal{A}_{0}} \frac{1}{2 d}>\frac{1}{2}
$$

Form this point on, the rest of the proof follows Yoccoz[20] almost verbatim. We include it here for completeness.

Step 3:

Since at $\pi_{\iota}$, a band outside $\mathcal{A}_{0}$ is in a critical position, it is not possible to get a stage in which both bands in the critical positions are in $\mathcal{A}_{0}$ unless a band in $\mathcal{A}_{0}$ moves a band outside. This observation can be exploited to show that with a definite probability, either a band outside $\mathcal{A}_{0}$ becomes large enough in norm or a band in $\mathcal{A}_{0}$ moves a band outside.

For each $\mathbf{x} \in X$, let $\pi_{\mathbf{x}, m}$ denote the stage after $\pi_{\iota}$ such that $\pi_{\mathbf{x}, m-1} \rightarrow \pi_{\mathbf{x}, m}$ is the first instance when a band in $\mathcal{A}_{0}$ is split. To each $\mathrm{x} \in X$, we associate a stage $\pi(\mathbf{x})$ in the path $\pi_{\iota} \rightarrow \cdots \rightarrow \pi_{\mathbf{x}, m}$ as follows: 
1. Case (1): If at some intermediate stage $\pi^{\prime}$

$$
\left|Q^{\prime}(\beta)\right| \geq \max _{\alpha \in S}\left|Q_{\iota}(\alpha)\right|
$$

for some $\beta \in S \backslash \mathcal{A}_{0}$, then we let $\pi(\mathbf{x})$ be the first instance when Inequality (8.0.25) is true for some band in $S \backslash \mathcal{A}_{0}$. This also means that in $\pi(\mathbf{x})$, there is a unique band $\beta \in S \backslash \mathcal{A}_{0}$ that satisfies Inequality (8.0.25).

2. Case (2): If no band in $S \backslash \mathcal{A}_{0}$ satisfies Inequality (8.0.25) at any intermediate stage, we set $\pi(\mathbf{x})=\pi_{\mathbf{x}, m}$. Here a band $\beta \in S \backslash \mathcal{A}_{0}$ splits a band $\alpha \in \mathcal{A}_{0}$ in the final split before $\pi(\mathbf{x})$. So

$$
\left|Q_{\theta}(\beta)\right|=\left|Q_{\theta-1}(\beta)\right|+\left|Q_{\theta-1}(\alpha)\right|
$$

Step 4:

Given the estimate (8.0.24), it is possible to select a finite number of stages $\pi_{\theta}$ from the collection of stages $\pi(\mathbf{x})$ constructed in Step 3 such that $J Q_{\theta}\left(W_{\theta}\right)$ have disjoint interiors and

$$
\ell\left(\cup_{\theta} J Q_{\theta}\left(W_{\theta}\right)\right)>\frac{1}{2} \ell(X)>\frac{1}{4} \ell\left(J Q_{\iota}\left(W_{\iota}\right)\right)
$$

For each $\pi_{\theta}$ as above, let $\mathcal{A}$ be the set of $\gamma \in S$ that satisfy

$$
\left|Q_{\theta}(\gamma)\right|>C_{0}^{-1} \max _{\alpha \in \mathcal{A}_{0}}|Q(\alpha)|
$$

Claim 8.0.28. For each $\theta$, the collection $\mathcal{A}$ is strictly larger than $\mathcal{A}_{0}$ and is $C_{0} C_{1}^{\prime}(1+M)$-distributed.

Proof. The stage $\pi_{\theta}$ belongs to one of the two cases in Step 3. In both cases, 
the distinguished band $\beta$ is in $\mathcal{A} \backslash \mathcal{A}_{0}$.

Given the lower bound (8.0.27) in the definition of $\mathcal{A}$, it is enough to show that the column largest in norm has an appropriate upper bound. Because we are dealing with subsets of $X$, every band $\alpha \in \mathcal{A}_{0}$ satisfies

$$
\left|Q_{\theta}(\alpha)\right|<M\left|Q_{\iota}(\alpha)\right|
$$

This implies

$$
\max _{\alpha \in \mathcal{A}_{0}}\left|Q_{\theta}(\alpha)\right|<M \max _{\alpha \in \mathcal{A}_{0}}\left|Q_{\iota}(\alpha)\right|
$$

If the largest column at $\pi_{\theta}$ is in $\mathcal{A}_{0}$, then estimates (8.0.22), (8.0.27) and (8.0.30) imply that $\mathcal{A}$ is $C_{0} C_{1}^{\prime} M$-distributed. So it is also $C_{0} C_{1}^{\prime}(1+M)$-distributed and we are done. Hence to finish the proof, we shall assume that the largest column is not in $\mathcal{A}_{0}$. A stage $\pi_{\theta}$ can belong to Case (1) or Case (2) of Step 3. We argue the two cases separately:

1. Case 1: If $\pi_{\theta}$ belongs to Case (1), then there is a unique band $\beta \in S \backslash \mathcal{A}_{0}$ that satisfies Inequality (8.0.25). By assumption, the largest column is not in $\mathcal{A}_{0}$; hence the column $Q_{\theta}(\beta)$ has to be the largest. At every stage before $\pi_{\theta}$, no column in $S \backslash \mathcal{A}_{0}$ satisfies Inequality (8.0.25) and no column in $\mathcal{A}_{0}$ moves a column in $S \backslash \mathcal{A}_{0}$. So we get the bound

$$
\left|Q_{\theta}(\beta)\right|<2 \max _{\alpha \in \mathcal{A}_{0}}\left|Q_{\iota}(\alpha)\right|<(1+M) \max _{\alpha \in \mathcal{A}_{0}}\left|Q_{\iota}(\alpha)\right|
$$

Combining with Inequality (8.0.22) results in the upper bound

$$
\left|Q_{\theta}(\beta)\right|<(1+M) \max _{\alpha \in \mathcal{A}_{0}}\left|Q_{\iota}(\alpha)\right|<C_{1}^{\prime}(1+M) \max _{\alpha \in \mathcal{A}_{0}}|Q(\alpha)|
$$

and so the collection $\mathcal{A}$ is $C_{0} C_{1}^{\prime}(1+M)$-distributed. 
2. Case 2: If $\pi_{\theta}$ belongs to Case (2), then in the final split before $\pi_{\theta}$, some band $\alpha$ in $\mathcal{A}_{0}$ is split by some band $\beta$ in $S \backslash \mathcal{A}_{0}$. So it satisfies (8.0.26). Along the sequence $\pi_{\iota} \rightarrow \cdots \rightarrow \pi_{\theta}$, no band in $S \backslash \mathcal{A}_{0}$ satisfies Inequality (8.0.25) at any intermediate stage. This implies that the column $Q_{\theta}(\beta)$ has to be the largest in norm. It also implies that

$$
\left|Q_{\theta-1}(\beta)\right|<\max _{\alpha \in \mathcal{A}_{0}}\left|Q_{\iota}(\alpha)\right|
$$

Inequalities (8.0.22) and (8.0.31) give the upper bound

$$
\left|Q_{\theta}(\beta)\right|<(1+M) \max _{\alpha \in \mathcal{A}_{0}}\left|Q_{\iota}(\alpha)\right|<C_{1}^{\prime}(1+M) \max _{\alpha \in \mathcal{A}_{0}}|Q(\alpha)|
$$

and so the collection $\mathcal{A}$ is $C_{0} C_{1}^{\prime}(1+M)$-distributed.

Setting $c_{1}=(1 / 4) c_{1}^{\prime}$ and $C_{1}=C_{0} C_{1}^{\prime}(1+M)$ concludes the proof of the Proposition 8.0.21.

Iterating Proposition 8.0.21, we get

Proposition 8.0.33. There exist constants $c \in(0,1)$ and $C>1$ that depend only on $d$, and a finite number of future stages $\pi_{\tau}$ after $\pi$, such that

1. The sets $J Q_{\tau}\left(W_{\tau}\right)$ have disjoint interiors and

$$
\sum_{\tau} \ell\left(J Q_{\tau}\left(W_{\tau}\right)\right) \geq c \cdot \ell(J Q(W))
$$

2. Each stage $\pi_{\tau}$ is $C$-distributed 
To prove Theorem 4.3.2, apply Proposition 8.0.33 to $\pi$ to get a set of stages, which we now denote by $\pi_{\tau}^{(1)}$, that are $C$-distributed. Let $X^{(1)}$ be the union of the sets $J Q_{\tau}\left(W_{\tau}\right)$. Consider the complement $J Q(W) \backslash X^{(1)}$. Write it as a union of stages with disjoint interiors. Apply Proposition 8.0.33 to each of them to get a new set of stages, which we denote by $\pi_{\tau}^{(2)}$, that are $C$-distributed. Let $X^{(2)}$ denote the union of the sets that correspond to the new $C$-distributed stages. Now consider the complement $J Q(W) \backslash X^{(1)} \cup X^{(2)}$ and iterate the process.

As a result, we construct an infinite sequence of sets $X^{(n)}$ with pairwise intersections of measure zero, such that they are a union of $C$-distributed stages. The set of $\mathbf{x} \in J Q(W)$ whose expansion gets $C$-distributed at some stage after $\pi$, is equal to the infinite union of the sets $X^{(n)}$. Let

$$
Y^{(n)}=J Q(W) \backslash \bigcup_{i=1}^{n-1} X^{(i)}
$$

By Proposition 8.0.33, we know that $\ell\left(Y^{(n)}\right) \leq(1-c) \ell\left(Y^{(n-1)}\right)$. Hence by induction

$$
\lim _{n \rightarrow \infty} \ell\left(Y^{(n)}\right)=0
$$

So the infinite union of the sets $X^{(n)}$ has full measure which concludes the proof of Theorem 4.3.2. An immediate consequence of Theorem 4.3 .2 is

Theorem 8.0.34. For almost every $\mathbf{x} \in W_{0}$, the expansion of $\mathbf{x}$ becomes C-distributed infinitely often.

Proof. Theorem 4.3.2 says that the set whose expansion never gets $C$-distributed is measure zero. The set of $\mathbf{x}$ whose expansion gets $C$-distributed finitely many times is a countable union of such measure zero sets. Hence it is measure zero, proving the theorem. 


\section{Chapter 9}

\section{Normality}

In this section, we prove normality, i.e., Theorem 1.0.2. We now clarify the phrase "non transient sequences that can occur" in the statement of Theorem 1.0.2. Recall that to avoid transient vertices, we assumed that the initial stage $\pi_{0}$ is in some $\operatorname{sink} G_{0}$ of the reduced diagram $G$. So a non-transient sequence that can occur is any finite sequence in $G_{0}$.

For every $\pi^{\prime}$ in $G_{0}$, choose a shortest splitting sequence from $\pi^{\prime}$ to $\pi_{0}$. This gives a finite number of chosen splitting sequences. This means that for any $C$-distributed $\pi^{\prime}$

1. With probability bounded below by a constant $c^{\prime}>0$ that depends only on $d$, it is possible to split from the $C$-distributed $\pi^{\prime}$ to a stage that is combinatorially $\pi_{0}$ using the chosen splitting sequence.

2. Splitting from $\pi^{\prime}$ to a stage as above, introduces a bounded amount of distortion in the measure, where the bound depends only on $d$.

The statements above imply that in Proposition 8.0.33, for different constants

$c \in(0,1)$ and $C>1$ which depend only on $d$, one gets $C$-distributed with a relative probability greater than $c$, such that the $C$-distributed stages are combinatorially $\pi_{0}$. In other words, the uniformly distorted stages in Theorem 4.3.2 can be assumed to be combinatorially $\pi_{0}$. Moreover, because of this 
additional conclusion in Theorem 4.3.2 and since $G_{0}$ is strongly connected in the directed sense, it is enough to prove normality for finite sequences that start from $\pi_{0}$.

Let $\jmath=\pi_{0} \rightarrow \cdots \rightarrow \pi$ be a finite sequence of splits. We prove the stronger form of normality stated below, which is necessary to prove unique ergodicity.

Theorem 9.0.1 (Strong Normality). In almost every expansion, for any finite sequence $\mathrm{J}$ starting from $\pi_{0}$, there are infinitely many instances in which $\mathrm{J}$ immediately follows a C-distributed stage.

Proof. By Theorem 8.0.34, for every $k \in \mathbb{N}$, there is a $k$-th instance of $C$ distribution in the expansion of almost every $\mathbf{x} \in W_{0}$. Let $\pi_{\mathbf{x}, n_{k}}$ be the $C$ distributed stage that is the $k$-th instance. For a finite non-transient sequence J, let $X_{k}$ denote the set of all $\mathbf{x}$ for which the expansion after $\pi_{\mathbf{x}, n_{k}}$ begins with . Recall from Chapter 6 , that a $C$-distributed stage is $C^{d-1}$-uniformly distorted. So the probability that $\jmath$ follows any $C$-distributed stage is, up to a constant that depends only on $C$ and $d$, the same as the probability that an expansion begins with $\jmath$. Let this probability be $\mu>0$. So we have $\ell\left(X_{k}\right) \approx \mu$. Since

$$
\sum_{k=1}^{\infty} \ell\left(X_{k}\right)=\infty
$$

by the standard Borel-Cantelli Lemma [18], the set of $\mathbf{x}$ that belong to infinitely many $X_{k}$ has full measure. 


\section{Chapter 10}

\section{Unique Ergodicity}

By the first proposition in Section 4.4 of Yoccoz [20], for every $\mathbf{x}$, the infinite intersection of the nested sequence of sets given by the expansion of $\mathbf{x}$ can be identified with the set of transverse measures. Consequently, to show unique ergodicity it is enough to show

Theorem 10.0.1. For almost every $\mathbf{x}$ in $W_{0}$, the nested sequence of sets $J Q_{n}\left(W_{n}\right)$ given by the expansion of $\mathbf{x}$ satisfy

$$
\bigcap J Q_{n}\left(W_{n}\right)=\mathbf{x}
$$

In other words, almost surely the infinite intersection nests down to a point.

Proof. It is enough to prove that almost surely, the sets $J Q_{n}(\Delta)$ nest down to a point. To do this, suppose there exists a finite sequence $\jmath: \pi_{0} \rightarrow \cdots \rightarrow \pi_{j}$ with the diameter shrinking property: There exists a universal positive constant $R<1$ such that when any $C$-distributed stage $\pi$ is followed by $\jmath$, the diameter becomes less than the diameter at $\pi$ by a factor less than $R$. By strong normality, in almost every expansion, the sequence $\jmath$ follows a $C$-distributed stage infinitely often. This implies that for almost every $\mathbf{x}$, the diameter shrinking occurs infinitely often and so the infinite intersection of the sets $J Q_{n}(\Delta)$ has diameter zero, i.e., it is a point. 
It is not immediate from the definition of $C$-distribution that a sequence J that shrinks diameter by $R<1$ at $W_{0}$ also shrinks diameter at every $C$ distributed stage by $R$ (up to a constant that depends only on $C$ and $d$ ), i.e., that $\mathrm{J}$ has the diameter shrinking property. The subtle issue is that a $C$-distributed stage is defined using the Jacobian. So it is not immediate that distortion is uniform along line segments in $\Delta$. The following lemma shows that for a $C$-distributed stage, the distortion along line segments in $\Delta$ is $C^{2}$ uniform.

Let $\pi$ be a $C$-distributed stage and let $Q$ be the associated matrix. Let $L$ be a line segment in $\Delta$ with endpoints $\mathbf{u}_{1}$ and $\mathbf{u}_{2}$ in $\partial \Delta$. Denote the unit tangent vector along $L$ from $\mathbf{u}_{1}$ to $\mathbf{u}_{2}$ by $v$. Let $\mathbf{w}_{1}=Q \mathbf{u}_{1}$ and $\mathbf{w}_{2}=Q \mathbf{u}_{2}$. As in Chapter 6 , denote the full derivative of $J Q$ as a map from $\Delta$ to itself by DJQ.

Lemma 10.0.2. For any pair of points $\mathbf{y}_{1}$ and $\mathbf{y}_{2}$ in $L$,

$$
\frac{1}{C^{2}}\left|\mathbf{D} J Q\left(\mathbf{y}_{1}\right)(v)\right|<\left|\mathbf{D} J Q\left(\mathbf{y}_{2}\right)(v)\right|<C^{2}\left|\mathbf{D} J Q\left(\mathbf{y}_{1}\right)(v)\right|
$$

Proof. Parameterize $Q(L)$ by $f(t)=(1-t) \mathbf{w}_{1}+t \mathbf{w}_{2}$. Then $g(t)=f(t) /|f(t)|$ is a parametrization of $J Q(L)$. By additivity of the norm, $|f(t)|=(1-t)\left|\mathbf{w}_{1}\right|+$ $t\left|\mathbf{w}_{2}\right|$. Computing the derivative with respect to $t$, we get $|f(t)|^{\prime}=\left|\mathbf{w}_{2}\right|-\left|\mathbf{w}_{1}\right|$ This gives

$$
\begin{aligned}
g^{\prime}(t) & =\frac{|f(t)| f^{\prime}(t)-f(t)|f(t)|^{\prime}}{|f(t)|^{2}} \\
& =\frac{\left((1-t)\left|\mathbf{w}_{2}\right|+t\left|\mathbf{w}_{1}\right|\right)\left(\mathbf{w}_{2}-\mathbf{w}_{1}\right)-\left((1-t) \mathbf{w}_{1}+t \mathbf{w}_{2}\right)\left(\left|\mathbf{w}_{2}\right|-\left|\mathbf{w}_{1}\right|\right)}{|f(t)|^{2}} \\
& =\frac{\left|\mathbf{w}_{1}\right| \mathbf{w}_{2}-\left|\mathbf{w}_{2}\right| \mathbf{w}_{1}}{|f(t)|^{2}}
\end{aligned}
$$


This means that for any pair of values $t_{1}$ and $t_{2}$ in the interval $[0,1]$,

$$
\frac{1}{C^{2}}<\frac{\left|g^{\prime}\left(t_{1}\right)\right|}{\left|g^{\prime}\left(t_{2}\right)\right|}<C^{2}
$$

Let $h(t)=(1-t) \mathbf{u}_{1}+t \mathbf{u}_{2}$ parameterize $L$. The map $J Q$ restricted to $L$ is given by $J Q(\mathbf{y})=g \circ h^{-1}(\mathbf{y})$. Since $h$ is a parametrization of $L$ with constant speed, the estimate (10.0.3) concludes the proof.

By Corollary 4 from Section 4.3 of Yoccoz [20], there exists a splitting sequence $\jmath$ from $\pi_{0}$ whose associated matrix has every entry positive. For nonclassical interval exchanges, the corollary is a consequence of Proposition 8.0.1 using an argument identical to the original proof in Yoccoz. The corollary implies that the image of $\Delta$ under the associated map is in the interior of $\Delta$. We fix this sequence $\jmath$ for the rest of this section.

Let $t_{\alpha}$ be the vertices $J Q_{\jmath}(\alpha)$ of $J Q_{\jmath}(\Delta)$. Let $s$ denote the minimum distance between $t_{\alpha}$ and $\partial \Delta$. The number $s$ is related to the largest entry of $Q_{\jmath}$.

Extend the edge $\left[t_{\alpha}, t_{\beta}\right]$ of $J Q_{\jmath}(\alpha)$ in either direction to a line segment $L[\alpha, \beta]$ with corresponding endpoints $u_{\alpha}$ and $u_{\beta}$ in $\partial \Delta$. Then the distances $d\left(t_{\alpha}, u_{\alpha}\right)$ and $d\left(t_{\beta}, u_{\beta}\right)$ are greater than $s$. So the length of $\left[t_{\alpha}, t_{\beta}\right]$ is at most $(1-2 s)$ times the length of $L[\alpha, \beta]$.

Proposition 10.0.4. Suppose $\pi$ is a $C$-distributed stage and let $\pi^{\prime}$ be the stage obtained by $\mathrm{J}$ following $\pi$. Then there exists a positive constant $R<1$ that depends on $s, C$ and $d$ such that

$$
\operatorname{diam}\left(J Q^{\prime}(\Delta)\right)<R \operatorname{diam}(J Q(\Delta))
$$

Proof. Since $J Q^{\prime}(\Delta)$ is a simplex, the longest line segment in it is a side joining 
a pair of vertices. Since $Q^{\prime}=Q \cdot Q_{j}$, we assume that this side is $J Q\left(\left[t_{\alpha}, t_{\beta}\right]\right)$. Let $v$ be the unit tangent vector along the segment $L[\alpha, \beta]$. By Lemma 10.0.2, the distortion of $v$ is $C^{2}$-uniform on $L[\alpha, \beta]$. Combining this with the fact that the length of $\left[t_{\alpha}, t_{\beta}\right]$ is at most $(1-2 s)$ times the length of $L[\alpha, \beta]$, we get the distance estimate

$$
\frac{d\left(J Q\left(t_{\alpha}\right), J Q\left(t_{\beta}\right)\right)}{d\left(J Q\left(u_{\alpha}\right), J Q\left(u_{\beta}\right)\right)}<\frac{C^{2}(1-2 s)}{C^{2}(1-2 s)+2 s}
$$

Taking $R$ to be the right hand side in the above inequality finishes the proof of Proposition 10.0.4.

Strong normality applied to the diameter shrinking sequence $\jmath$ implies that diameter shrinking happens infinitely often in almost every expansion, and so almost surely, the infinite intersection is a point.

Finally, Theorem 10.0.1 implies that for almost every $\mathbf{x}$, there is a unique transverse probability measure. Consequently, almost every non-classical interval exchange is uniquely ergodic. 


\section{Chapter 11}

\section{Rauzy Map on the Parameter Space}

Consider the full parameter space over a sink for a non-classical interval exchange. It is a disjoint union of configuration spaces, each associated to different combinatorial types in the sink. Rauzy induction induces a map from this parameter space to itself described in Chapter 4. Each configuration space in the full parameter space carries a natural Lebesgue measure. A consequence of Theorems 8.0.34 and 10.0.1 is

Theorem 11.0.1. The Lebesgue measure on the full parameter space is ergodic with respect to the Rauzy map.

Proof. Let $A$ be a Borel set in the full parameter space invariant under the Rauzy map and suppose that $A$ is not full measure. Then $A$ is not full measure in some configuration space $W$ corresponding to the combinatorial type $\pi$. Let $\mathbf{x}$ be a point of Lebesgue density for the complement $W \backslash A$. Given $1 / 2>\epsilon>0$, there is a ball $B(\mathbf{x})$ centered at $\mathbf{x}$ such that the proportion of $A$ in this ball is less than $\epsilon$. By Theorems 8.0.34 and 10.0.1, the ball $B(\mathbf{x})$ can be closely approximated by a union of $C$-distributed stages contained in $B(\mathbf{x})$. Let us suppose the approximation is chosen so that the union of these $C$-distributed stages has measure greater than $(1-\epsilon) \ell(B(\mathbf{x}))$. If the proportion of $A$ in each 
of these $C$-distributed stages were greater than $2 \epsilon$, then that would imply that the proportion of $A$ in $B(\mathbf{x})$ is greater than $2 \epsilon(1-\epsilon)>\epsilon$, a contradiction. This means that the proportion of $A$ in at least one of the $C$-distributed stages is at most $2 \epsilon$. Call this stage $T$.

Let $\pi^{\prime}$ be any combinatorial type in the sink, and let $W^{\prime}$ be the associated configuration space. Due to the strong connectivity of the sink, it may be assumed that $T$ is actually obtained by a splitting sequence $\int$ starting at $\pi$ and terminating in $\pi^{\prime}$.

By the invariance of $A$, the proportion of $A$ in $W^{\prime}$ is at most $2 \epsilon$. Since $\epsilon$ can be made arbitrarily small and $\pi^{\prime}$ is any combinatorial type in the sink, the conclusion is that $A$ must have measure zero. Thus the only invariant sets for the Rauzy induction on the parameter space associated to a sink have zero or full measure, i.e., the Rauzy map is ergodic. 


\section{Chapter 12}

\section{Linear Involutions}

Non-classical interval exchanges are linear involutions [6] that correspond to the principle stratum of quadratic differentials. In [6], Boissy and Lanneau establish criteria for irreducibility of linear involutions. These criteria indicate when a particular linear involution either sits in a sink or always splits to end up in some sink. They give two notions for irreducibility of linear involutions: geometric and dynamical. The geometric notion of irreducibility is the strongest and implies dynamical irreducibility. It also implies that the Veech construction for suspending the linear involution holds and that the orbits for almost all starting widths are minimal. Moreover, a geometrically irreducible linear involution always belongs to some sink for the associated Rauzy diagram. The sinks are called Rauzy classes in literature and they classify the connected components of the associated stratum of quadratic differentials.

On the other hand, dynamical irreducibility is a weaker notion and does not define a Rauzy class unless the width data comes from a suitably restricted set called the admissible set. In many situations, the admissible set has full

measure. But there are instances when this is not so. It turns out that a linear involution that is dynamically irreducible and has admissible widths eventually splits down to a linear involution that is geometrically irreducible. More importantly for us, a dynamical irreducible linear involution that has 
admissible widths is also measure irreducible in our sense. In particular, this means that if we restrict to some sink, any geometrically irreducible involution that sits in it, is measure irreducible in our sense.

To prove the theorems for non-classical interval exchanges, the logical correlations we used were as follows: Completeness of the underlying train tracks implies measure irreducibility, measure irreducibility implies Proposition 8.0.1, and finally Proposition 8.0.1 combined with the analysis of the restriction of the Jacobian gives the proofs of the main theorems.

So to generalize the results to linear involutions, it is enough to restrict to some sink in the associated Rauzy diagram. Then the linear involutions considered are geometrically irreducible and hence measure irreducible. Consequently, all the theorems are true for geometrically irreducible linear involutions. 


\section{Chapter 13}

\section{Applications and Future Research}

\subsection{Harmonic measures on $\mathcal{P} \mathcal{M} \mathcal{F}$}

The mapping class group $G$ of an oriented closed surface $\Sigma$ is the group of orientation preserving diffeomorphisms of the surface modulo those isotopic to identity. An oriented surface can be given a conformal structure making it a Riemann surface. The Teichmüller space $T(\Sigma)$ is the space of all marked conformal structures modulo biholomorphisms isotopic to identity. By the uniformization theorem, up to isometries isotopic to identity, there is a unique marked hyperbolic metric on $\Sigma$ in each marked conformal class. Hence the Teichmüller space $T(\Sigma)$ can also be thought of as the space of marked hyperbolic metrics on the surface modulo isometries isotopic to identity. The mapping class group $G$ acts on $T(\Sigma)$ by changing the marking, and the quotient is called the moduli space of curves.

Topologically, the space $T(\Sigma)$ is homeomorphic to an open ball in $\mathbb{R}^{6 g-6}$, where $g$ is the genus of the surface. Thurston showed that $T(\Sigma)$ can be naturally compactified by the space $\mathcal{P} \mathcal{M} \mathcal{F}$ of projective measured foliations on $\Sigma$, such that the action of $G$ extends continuously to the boundary. The space $\mathcal{P} \mathcal{M} \mathcal{F}$ is homeomorphic to the sphere $S^{6 g-7}$. 
In [11], Kaimanovich and Masur considered random walks on $G$ with respect to any initial distribution $\mu$. It is possible to project the random walk into $T(\Sigma)$, by choosing a base-point and then using the action of $G$. They showed that if the initial distribution $\mu$ has finite moment, and the subgroup generated by the support of $\mu$ is non-elementary, i.e., contains two pseudo-Anosov elements with distinct stable and unstable foliations, then almost every sample path for the random walk converges to a point in $\mathcal{P} \mathcal{M} \mathcal{F}$. This means that there is a well defined hitting measure $\nu$ on $\mathcal{P} \mathcal{M} \mathcal{F}$ coming from the random walk. The measure $\nu$ is harmonic in the sense that if $A$ is a measurable set of $\mathcal{P \mathcal { F }}$

$$
\nu(A)=\sum_{g \in A} \mu(g) \nu\left(g^{-1} A\right)
$$

,,i.e. ,,the measure of a set is equal to the weighted average of its translates.

The space $\mathcal{P} \mathcal{M} \mathcal{F}$ carries a natural Lebesgue measure class. For instance, the parameter space associated to a complete train track on the surface determines a chart on $\mathcal{P} \mathcal{M} \mathcal{F}$. The natural volume form on the parameter space induces a measure on this chart that is in the Lebesgue measure class.

In [10], I used Theorem 4.3.2 to show the following theorem:

Theorem 13.1.1. If $\mu$ is a finitely supported initial probability distribution on $G$ such that the subgroup of $G$ generated by the support of $\mu$ is non-elementary, then the induced harmonic measure $\nu$ on $\mathcal{P} \mathcal{M} \mathcal{F}$ is singular with respect to the Lebesgue measure class.

To prove Theorem 13.1.1, we construct a singular set by using a modified Borel-Cantelli Lemma and it's converse applied to an appropriate sequence of "cylinder" sets for the symbolic dynamics given by splitting sequences of nonclassical interval exchanges. For the Lebesgue measure part, Theorem 4.3.2 gives us the key control to estimate the Lebesgue measure of these cylinder 
sets. For the harmonic measure part, we use a theorem due to Maher that shows exponential decay for the harmonic measure for nesting with respect to some sub-surface projection.

\subsection{Future directions}

The theory of classical interval exchanges is rich. A non-classical interval exchange or more generally, a geometrically irreducible linear involution can be interpreted as a measure preserving map $f$ from a pair of disjoint intervals into itself. It would be interesting to know which results that hold for classical interval exchanges generalize to geometrically irreducible linear involutions.

For instance, Avila and Forni [3] showed that almost every irreducible class interval exchange is weakly mixing. Moreover, a classical interval exchange can be suspended using the zippered rectangles construction due to Veech [19]. This relates interval exchanges to Teichmüller flow on the space of abelian differentials. Using the fast Rauzy induction due to Zorich, it was shown independently by Athreya [1], by Bufetov [7] and by Avila, Gouezel and Yoccoz [4] that the Teichmüller flow is exponentially mixing. As mentioned in [4], this makes the proof of weak mixing more transparent. One can then ask:

Question 13.2.1. Is the above map weakly mixing for almost all geometrically irreducible linear involutions?

Similar to the classical interval exchanges, it is possible to suspend the map to relate it to the Teichmüller flow on a stratum of quadratic differentials. Recently, Avila and Resende [5] have shown exponential mixing for the Teichmüller flow on quadratic differentials. I am currently investigating the possibility that their work may have applications to Question 13.2.1. 


\section{Bibliography}

[1] Athreya, J. Quantitative recurrence and large deviations for Teichmüller geodesic flow, Geom. Dedicata 119 (2006), 121-140.

[2] Avial, A. and Bufetov, A. Exponential decay of correlations for RauzyVeech-Zorich induction map, Partially hyperbolic dynamics, laminations, and Teichmüller flow, 203-211, Fields Ins. Comm. 51, Amer. Math. Soc, Providence, RI 2007.

[3] Avila, A. and Forni, G. Weak mixing for interval exchange transformations and translation flows, Ann. of Math. (2) 165 (2007), 2, 637-664.

[4] Avila, A., Gouëzel, S. and Yoccoz, J. Exponential mixing for the Teichmüller flow, Publ. Math. Inst. Hautes Études Sci. 104 (2006) 143-211.

[5] Avila, A. and Resende, M. Exponential mixing for the Teichmüller flow in the space of quadratic differentials, http://arxiv.org/abs/0908.1102

[6] Boissy, C. and Lanneau, E. Dynamics and Geometry of the Rauzy-Veech induction for quadratic differentials, Ergodic Theory and Dynamical Systems 29 (2009), no 3, 767-816.

[7] Bufetov, A. Decay of correlations for the Rauzy-Veech-Zorich induction map on the space of interval exchange transformations and the central limit theorem for the Teichmüller flow on the moduli space of abelian differentials, J. Amer. Math. Soc 19 (2006), no 3, 579-623. 
[8] Danthony, C. and Nogueira, A. Measured foliations on non-orientable surfaces, Ann. Sci. Ecole. Norm. Sup (4) 23 (1990), 3, 469-494.

[9] Dunfield, N. and Thurston, D. A random tunnel number one 3-manifold does not fiber over the circle, Geometry and Topology 10 (2006), 24312499.

[10] Gadre, V. Harmonic measures for distributions with finite support on the mapping class group are singular, http://arxiv.org/abs/0911.2891.

[11] Kaimanovich, V.A. and Masur, H. The Poisson boundary of the mapping class group, Inventiones Mathematicae (1996) 125, 221-264.

[12] Kaimanovich, V.A. and Masur, H. The Poisson boundary of Teichmuller space, Journal of Functional Analysis 156 (1998), 301-332.

[13] Kerckhoff, S.P. Simplicial systems for interval exchange maps and measured foliations, Ergodic Theory and Dynamical Systems (1985) 5, 257271.

[14] Masur, H. Interval exchange transformations and measured foliations, Ann. of Math. (2) 115 (1982), no 1, 169-200.

[15] Nogueira, A. Almost all interval exchange transformations with flips are non-ergodic, Ergodic Theory and Dynamical Systems 9 (1989), 3, 515-525.

[16] Penner, R. with Harer, J. Combinatorics of train tracks, Princeton University Press (1992).

[17] Rees, M. An alternative approach to the ergodic theory of measured foliations on surfaces Ergodic Theory and Dynamical Systems (1981) 1, 461-488. 
[18] Stroock, D. Probability Theory, An analytic view, Cambridge University Press (1993).

[19] Veech, W. Guass measures for transformations on the space of interval exchange maps, Ann. of Math. (2) 115 (1982), no 1, 201-242.

[20] Yoccoz, J-C. Continued Fraction Algorithms for Interval Exchange Maps: an Introduction, Frontiers in Number Theory, Geometry and Physics I (2006), 401-435, Springer, Berlin.

[21] Zorich, A. Finite Gauss measure on the space of interval exchange transformations. Lyapunov exponents, Annales de L'Institut Fourier, Tome 46, fasc. 2, (1996), 325-370. 